\title{
Prekäres Erzählen und das Ordnungsproblem der Moderne. Einleitung
}

\begin{abstract}
Simon war zwanzig Jahre alt, als ihm eines Abends in den Sinn kam, er könnte so, wie er gerade im weichen, grünen Moose am Wege lag, fortwandern und Page werden. Dies sprach er sehr laut in die Luft hinauf zu den Tannengipfeln, welche, ich weiß nicht ob es wahr oder erlogen ist, ihre scheinheiligen Bärte schüttelten und ein stummes, tannzapfenartiges Gelächter anstimmten, welches unserem Mann auf die Beine half und ihn antrieb, sofort das zu werden, wozu ihn eine unbändige Lust anfeuerte. Jetzt hat er sich erhoben und marschiert ins Blaue oder Grüne hinein [...]. Kümmern wir uns ein wenig um sein Äußeres! (SW 2, 15) ${ }^{1}$
\end{abstract}

Dies ist der Anfang der Erzählung Simon. Eine Liebesgeschichte, die Robert Walser 1904 in der Münchner Zeitschrift Die Freistatt publizierte und im 1914 erschienenen Band Geschichten wiederabgedruckt hatte. ${ }^{2}$ Simon wandert in die Welt hinein: Vom epischen Präteritum der ersten Sätze („Simon war zwanzig Jahre alt“) über die Mittelstellung des Perfekt („hat er sich erhoben“) marschiert Simon zielstrebig in die Gegenwart des Präsens („marschiert ins Blaue oder Grüne“) hinein. Den Anfang seiner Geschichte erzählt Simon gleich selbst, indem er sie „sehr laut in die Luft hinauf“ zu den Bäumen spricht. Der Wunsch, Page zu werden, wird dadurch verwirklicht, dass Simon sich auf der Buchseite, der französischen page, manifestiert. Die Tannen als Figurationen des Bleistifts tragen das ihre dazu bei. Mit der Wendung „ins Blaue oder Grüne hinein" wird schon hier auf die Spannung zwischen innerfiktionaler Wirklichkeit - in der das Grüne den Wald bezeichnet - und der Ebene des Erzählens - ins Blaue hinaus, also ohne Ziel und doch auch mit Hinweis auf den Blaustift als Korrekturzeichen - verwiesen. ${ }^{3}$ Damit kommt das Erzählen dezidiert in den Blick.

1 Im Folgenden wird unter der Sigle SW zitiert nach: Robert Walser, Sämtliche Werke in Einzelausgaben, hg. von Jochen Greven, 20 Bde. (Zürich, Frankfurt am Main: Suhrkamp, 1985f.).

2 Robert Walser, „Simon. Eine Liebesgeschichte“, Freistatt. Süddeutsche Wochenschrift für Politik, Literatur und Kunst (München) 6, Nr. 14 (2. April 1904): 266-267.

Folgende Ausführungen wurden in ähnlicher Form publiziert: Lukas Gloor, „Robert Walsers unordentliche Ordnung in ,Simon. Eine Liebesgeschichte“", in Goldenes Anfängliches. Neue Beiträge zur Robert Walser-Forschung, hg. von Lukas Gloor und Rebecca Lötscher, Robert Walser-Studien 4 (Paderborn: Wilhelm Fink, 2020), 133-143.

3 Vgl. zur Bedeutung des Blaustifts in den Geschwister Tanner Ines Barner, „, Ich beneide dumme Jungens ganz rasend.' Zum Briefwechsel zwischen Robert Walser und Christian Morgenstern" (Vortrag, Jahrestagung der Robert Walser-Gesellschaft, Bern, 20. Oktober 2018).

(C) LUKAS GLOOR, 2020 | DOI:10.30965/9783846765593_002

This is an open access chapter distributed under the terms of the CC BY-NC-ND 4.o licenses Gloor - 9783846765593 
Dieses Erzählen bringt die Regeln des konventionellen Erzählmodells in Unordnung: Autor, Erzähler, Figur wie Erzählen und Erzähltes werden als Elemente zwar aufgeboten, aber nicht in der korrekten narratologischen Ordnung: Es taucht eine Figur Simon auf, die ihre Geschichte erzählt, und trotzdem gibt es einen Erzähler, der hier in Wir-Form, später auch aus der Ich-Perspektive sprechen wird. Die Erzählung wird einmal als Erzähler, einmal als streitbares Gegenüber des Erzählers bezeichnet und schließlich soll gar der Verfasser selbst auftreten. Nicht genug, dass die Erzählordnung formal durcheinandergeschüttelt wird, auch inhaltlich werden Ordnungsmodelle die Gattungen des Märchens und der Trivialgeschichte - zitiert, topografische Grenzen eingeführt und Marker von Genres eingesetzt, Rollenspiele aufgeführt, die Identitäten wiederum als sprachliche Konstruktionen darstellen. Simon. Eine Liebesgeschichte ist selbst eine Art Möbiusband des Erzählens. Durch die deutliche, stellenweise geradezu programmatische Thematisierung von Erzählordnungen eignet sich dieser frühe Text besonders, in diese Studie, die Analyse der narrativen Verfahren zur Herstellung und Subversion von Ordnung in der Moderne durch prekäres Erzählen, einzuführen.

Ziel dieser Studie ist es, das Spannungsfeld der Darstellungsweisen und Umgangsformen mit Ordnung in der Moderne anhand von Theodor Fontanes Der Stechlin (1898), Franz Kafkas Der Bau 4 (verfasst um 1923) und Texten Robert Walsers, hauptsächlich Der Spaziergang (1917/1919), der „Räuber"-Roman (verfasst um 1925) und einschlägigen Kurztexten, zu zeichnen. Leitend ist dabei der Begriff des prekären Erzählens, der dieser Studie den Titel gibt. Mit diesem Begriff können die Ordnungen der Texte wie die Darstellungen von Ordnungen in den Texten, mithin die narrativen Ordnungsleistungen der genannten Autoren mit einem eigenständigen Ausdruck beschrieben werden, anstatt das Defizit dieses Erzählens vor dem Hintergrund etablierter Erzählmodelle zu betonen. Es ist ein heuristisches Konzept, das den Zweck hat, den Blick für die Ordnungs- und Erzählthematik zu öffnen. Walser, Kafka und Fontane werden hier als implizite Ordnungstheoretiker und explizite Ordnungspraktiker verstanden, die sich dem Ordnungsproblem der Moderne aussetzen.

Diese Einleitung gliedert sich in einen ersten Teil, der mittels der Lektüre von Simon. Eine Liebesgeschichte den Komplex von Erzählen und Ordnung greifbar macht und den Begriff des prekären Erzählens skizziert. Im zweiten Teil wird mit der Diskussion der Moderne als Ordnungsproblem der kulturwissenschaftliche Hintergrund des prekären Erzählens erarbeitet. Damit

4 Der Titel Der Bau stammt von Max Brod, der Kafkas Erzählung aus dem Nachlass herausgegeben hat. Der Einfachheit halber wird nicht vom ,Bau'-Konvolut gesprochen, sondern wie andernorts üblich Brods Titel verwendet. 
einher geht eine Diskussion des zeitgenössischen Begriffs der Ordnung wie dessen Funktion in der Narratologie.

Im Folgenden soll zunächst mit der Fortsetzung der oben begonnenen Lektüre von Simon. Eine Liebesgeschichte in die Thematik und Methodik eingeführt werden. Ausgehend von einer detaillierten Textanalyse und close readings werden die Funktionen von Ordnung untersucht und erzähltheoretische Extrapolationen vorgenommen. Der Fokus auf Robert Walser in der Einleitung verdankt sich dem Umstand, dass bei diesem eine besonders intensive Auseinandersetzung mit dem Erzählen zu beobachten ist, sowie der Tatsache, dass er bisher wenig in das Blickfeld explizit narratologischer Studien geriet, obwohl er, wie zu sehen sein wird, zahlreiche Herausforderungen an die Theorie stellt. Entsprechend beansprucht das Kapitel zu Walser rund doppelt so viel Raum wie diejenigen zu Theodor Fontane und Franz Kafka.

Mit der Kontextualisierung der Moderne als Ordnungsproblem wird der Boden für die anschließenden textzentrierten Kapitel bereitet. Dazu werden die Begriffe der Moderne und der Ordnung umrissen und ihre Funktion in der Narratologie verortet. Mit Georg Simmel, Fritz Mauthner und anderen wird Ordnung in der Moderne schließlich als hochkomplexe, künstliche Konstruktion greifbar, in der Kausalität und Notwendigkeit in die Kontingenz überführt wird.

Was prekäres Erzählen meint und inwiefern es mit Ordnung als Begriff und Phänomen zu tun hat, lässt sich an der frühen Erzählung Robert Walsers erstaunlich umfassend darstellen. In Simon. Eine Liebesgeschichte wird die Erzählung als Modell von Ordnung mit ihren Bestandteilen und deren Funktionen vorgeführt. Die Geschichte handelt von Simon, der als Wanderer und Sänger durch die Wälder zieht, bis er bei einer märchenhaften Burg seine Gebieterin findet, die er schlussendlich auch gegen ihren früheren Geliebten zu verteidigen weiß. Strukturell ist Simon immer auch als Erzählung einer Erzählung angelegt. Kurz nach dem oben zitierten Anfang heißt es:

An Simons Rücken (wir, die Erzählung, gehen jetzt immer hinter ihm her) hängt eine alte, wüste Mandoline und wir sehen, wie er dieselbe in die Hand nimmt und darauf zu zupfen anfängt. (SW 2, 15)

An dieser Stelle wird die Erzählung als Erzähler eben dieser Erzählung eingeführt. Mit der Personifikation der Erzählung als homodiegetischem Erzähler, 
d. h. als am Geschehen teilhabende Figur, wird eine paradoxe Erzählsituation erzeugt. Die Erzählung stellt per Definition die Gesamtheit des Textes dar, ist also die abschließende diegetische Ebene. Hier aber wird die Erzählung Simon selbst zum Gegenstand dieser Erzählung, so dass ein "narrativer Kurzschluss“, eine Metalepse, entsteht. ${ }^{5}$ In dieser paradoxen Situation gibt es zwei Lektürestrategien, um die Ordnung wiederherzustellen. Es lässt sich eine weitere Erzählinstanz konstruieren, eine weitere Rahmengeschichte aufbauen, in der jemand erzählt, wie eine Erzählung erzählt, also hinter oder über dem „wir, die Erzählung" ein weiteres Erzähl-Ich positioniert ist. Die andere Strategie besteht darin, dieses „wir, die Erzählung“ als oberflächliche Spielerei abzutun, die keine Beachtung verdient. Beide Lesestrategien sind wenig befriedigend, da sie aus dem Irritierenden des Textes keinen interpretatorischen Gewinn ziehen. ${ }^{6}$ Es ist zwar durchaus ein Spiel, das Robert Walser treibt und das im Frühwerk an manchen Stellen noch aufgesetzt scheint. Und doch zeigt sich in diesem Spiel schon der Ansatz einer Konzeptualisierung von prekärem Erzählen, das sich im Verlauf seines Werks umso stärker im Textgewebe verwurzeln wird.

In Simon. Eine Liebesgeschichte scheint es tatsächlich mehrere Erzähler zu geben: einen namenlosen Erzähler, der einmal als Ich-Erzähler, einmal als Wir-Erzähler auftritt, die Erzählung und schließlich Simon selbst. Dieser lässt sich als Verkörperung des Erzählprinzips verstehen: Mit dem Losmarschieren Simons setzt sich auch die Erzählung in Gang.

Simon könnte tatsächlich auch aus Erzählungen, von Robert Walser oder von anderen Autoren, in die vorliegende Liebesgeschichte eingewandert sein. Nicht nur verweisen die „Tannengipfel“ und das Figurendreieck SimonKlara-Aggapaia auf Walsers 1906 verfassten und 1907 erschienenen Roman Geschwister Tanner. Der schwärmerische Jüngling, abgerissene Poet und romantische Taugenichts auf der Suche nach der Dame seines Herzens hat deutliche Bezüge zum Taugenichts Joseph von Eichendorffs. Vor allem aber ist Simon selbst Erzählung: Wie eingangs erwähnt entspricht sein Wunschberuf des Pagen in der französischen und auch, für Walser weniger relevant, der englischen Übersetzung der Buchseite. Diese Metapher wird, abermals in ihrer Bildlichkeit variierend, weitergeführt: Wie der Buchdeckel die Seiten eines Buchs abdeckt, wird Simon gegen oben von einem Hut abgeschlossen, dem „blecherne[n] Deckel“ einer „alten, rostigen Bratpfanne“ (SW 2, 15). Der

5 Werner Wolf, Ästhetische Illusion und Illusionsdurchbrechung in der Erzählkunst. Theorie und Geschichte mit Schwerpunkt auf englischem illusionsstörenden Erzählen (Tübingen: Max Niemeyer, 1993), $35^{8}$.

6 An dieser Stelle sei auf die unnatural narratology verwiesen, die gerade versucht, paradoxe, mithin unmögliche Texte nicht zu konventionalisieren, sondern in ihrer Widerständigkeit bestehen zu lassen. Siehe Kap. 5.3 Unnatural Narratology, S. 257ff. unten. 
Hut kommt „langsam in eine Form hinein, in die ihn unsorgfältige Behandlung und geringer Stoff mit der Zeit bringen müssen" $(\mathrm{SW} \mathrm{2,15)}$. Dies kann als Aussage interpretiert werden, dass die Figur des romantischen Taugenichts in bisherigen Werken unsorgfältig behandelt und keinem ergiebigen Stoff ausgesetzt wurde. Jedoch spielt diese Beschreibung auch auf das eigene, formbewusste Erzählen an, welches die Figur insofern unsorgfältig behandelt, als es keinen Wert auf in konventioneller Sicht stimmige Übergänge, Einführungen von Figuren und Handlungen sowie räumliche Verortungen legt.

Der Erzählvorgang wird immer wieder auch von der Mandoline, die schon im Eingangszitat gezupft wird, figuriert. Sie ist, passend zum Hut, „alt" und „wüst“ (SW 2, 15). Wenn das Spiel pausiert, pausiert auch die Erzählung: „Er [Simon] hört auf [Mandoline zu spielen] und wir haben Zeit, uns auf neuen Atem zu besinnen." (SW 2, 16) Als würde die Erzählung Simons tatsächlich pausieren, wechselt der Text in das Präteritum und schildert Simons Gedankengänge, ohne sie als solche durch Anführungszeichen oder typografische Merkmale auszuzeichnen: „Wie seltsam, dachte Simon [...], daß die Welt keine Pagen mehr hat. Hat sie denn etwa keine schönen, großen Frauenzimmer mehr? Wohl nicht, denn ich besinne mich [...]. Mit solchen Gedanken und Empfindungen brachte er es ein Stück Weg weiter." (SW 2, 16) Gegen Ende dieses Gedankenberichts kommt die Erzählung als Figur wieder zur Sprache:

\begin{abstract}
Simon griff wieder in die Mandoline, auf welcher er Zauberer war. Die Erzählung setzt sich hinten wieder auf einen Stein und horcht ganz verblüfft. Unterdessen gewinnt der Verfasser Zeit, auszuruhen.

Es ist ein mühseliges Geschäft, Geschichten zu erzählen. Immer hinter solch einem langbeinigen, mandolinenspielenden romantischen Bengel herlaufen und horchen, was er singt, denkt, fühlt und spricht. Und der rohe Schurke von Page läuft immer und wir müssen hinter ihm herlaufen, als ob wir wahrhaftig des Pagen Page wären. Hört weiter, geduldige Leser, wenn ihr noch Ohren habt, denn jetzt machen bald verschiedene Personen ihre untertänigsten Reverenzen. Es wird lustiger. (SW 2, 16f.)
\end{abstract}

Hier wird kein typischer Protagonist der Romantik geschildert und auch kein Autor nach romantischem Vorbild. Der "Verfasser" ist kein souveräner Erzeuger des Textes, sondern ein geplagter und gejagter Autor in einer ironisch zugespitzten Schreibszene. Mit ihm intensiviert sich die Komplikation zwischen Erzähler, Erzählung und Figur. Wie oben die Erzählung Objekt der Figur („wir, die Erzählung, gehen jetzt immer hinter ihm her") sein kann, so ist hier der Verfasser in einer weiteren Metalepse der Erzählung untergeordnet. Die Hierarchien des klassischen Erzählmodells eines realen Autors, der eine Geschichte schreibt, in der ein mehr oder weniger stark markierter Erzähler ein vergangenes Ereignis wiedergibt, werden zur Gänze ad absurdum geführt. 
Mit dem Verfasser hat auch der Leser seinen Auftritt. ${ }^{7}$ Diesen ,Lesern' wird, entsprechend der doppelten Konnotationen der Schriftlichkeit und Mündlichkeit, zugemutet, zu hören, statt zu lesen, wenn sie "noch Ohren“ haben. Der „Bengel“, zugleich Protagonist und Erzählung, von dem erzählt wird bzw. die erzählt, hält sich an keine Regeln.

Wird oben noch das Dogma der Wahrscheinlichkeitsbeglaubigung der Erzählung durch die Augenzeugenschaft des Erzählers bedient, so wird diese anschließend untergraben. Der Erzähler verliert seinen Protagonisten aus den Augen, weil dieser in die Gemäuer seiner Dame eingetreten ist. Stattdessen muss der Erzähler sich seiner Fantasie bedienen. Diese wird jedoch als genauso zuverlässig und glaubhaft dargestellt wie die Augenzeugenschaft. Vor dem Hintergrund des selbstreflexiven Erzählens ist dies nachvollziehbar; gleichzeitig untergräbt der metafiktionale Charakter die Augenzeugenschaft.

Der Verfasser grübelt nun aus seiner gequälten Phantasie hervor, was seine Augen nicht mehr sehen dürfen. Die Phantasie hat durchdringende Augen. Keine zehnmetrige Mauer, kein noch so schwarzer giftiger Schatten hemmt ihren Blick, der Mauern und Schatten wie ein Netz durchsieht. (SW 2, 18)

Fantasie wird hier mit der Metapher des Blicks umschrieben. Sie ermöglicht es nicht, zu imaginieren, was sich hinter der Mauer abspielt, sondern vermag hindurchzublicken. Gleichzeitig wird somit der Augenzeugenschaft als Beglaubigung des Erzählten das Fundament entzogen. Wie die Erzählordnung auf der Ebene ihrer Elemente Figur, Erzähler, Erzählung und Autor, wie die Poetik des Spazierens und Hörens, werden auch widersprüchliche Modelle der Beglaubigung des Erzählten aufgeboten.

\subsubsection{Performanz als Ordnungsverfahren}

In Simon. Eine Liebesgeschichte werden die Elemente der Erzählung fast wie in einem Baukasten auseinandergenommen und vorgeführt. Durch das Hervortreten der Bestandteile und deren Beziehung untereinander wird deutlich, dass eine Erzählung einer gewissen formalen wie inhaltlichen Ordnung zu folgen hat, wie der Text selbst mit der Referenz auf das Genre der „Liebesgeschichte“ deutlich macht. Es sorgt für Irritation und führt zu logischen Widersprüchen, wenn die Elemente der Erzählung, wie hier Erzählung, Erzähler, Verfasser und Figur scheinbar inkonsistent, zumindest nicht gemäß der ihnen im Modell zugedachten Funktionen entsprechend verwendet werden. Indem an einer Stelle die Erzählung selbst ihrer Figur hinterherläuft und sie an einer anderen

7 Der einfachen Lesart willen wird in dieser Arbeit das generische Maskulinum verwendet. Selbstverständlich sind alle Geschlechter gemeint. 
ihren Verfasser beschreibt, an einer dritten Stelle Erzähler und Erzählung über den Inhalt streiten - also jeweils zu Figuren der Erzählung werden, was ihrer Funktion nicht entspricht -, macht der Text deutlich, dass es hier um eine Selbstbeobachtung geht, an der die Thematik der Ordnung besonders deutlich wird.

Dieselbe Beobachtung gilt auch für die inhaltliche Ebene, auf der Motive der Romantik und Stereotypen des Groschenromans als Eckpfeiler des Settings dienen: Die angebetete Dame, der Kampf der Rivalen und die kindliche und schwärmerische Unschuld des Protagonisten sind Elemente dieser Genres. Dazu sind die Schauplätze des Waldes und der Burg sowie das Auftauchen des Teufels deutliche Markierungen einer Ordnungsstruktur. Auch die intertextuellen Bezüge oder "Reverenzen“ (SW 2, 17), wie Eichendorffs Aus dem Leben eines Taugenichts oder der pikareske Roman, ${ }^{8}$ bieten Ordnungsmuster auf. So deutlich diese Ordnungsparameter auch aufgerufen werden, so absurd ist ihr Nebeneinander und die anschließende Übertretung durch Ironisierung und Parodisierung. Sie werden zitiert, nicht um Ordnung herzustellen, sondern um Ordnung in eine spielerische Dynamik zu überführen und damit eine neue Ordnungsweise zu generieren. Indem Versatzstücke herkömmlicher Ordnungen inhaltlich kombiniert und formal subvertiert werden, wird gleichzeitig eine neue Ordnung der Performanz im Akt des Erzählens erzeugt.

Performanz spielt in Simon. Eine Liebesgeschichte auf verschiedenen Ebenen eine Rolle. In formaler Hinsicht wird durch zahlreiche metanarrative Kommentare und metaleptische Strukturen der Akt des Erzählens als Ursache des Erzählten hervorgehoben. Auf konzeptueller, poetologischer Ebene findet die Performanz ihren Ausdruck in der fast parodistischen Verwendung verschiedener Modelle zur Authentifizierung der Erzählung. Zum einen wird mit dem homodiegetischen Erzähler die Augenzeugenschaft und damit die Wahrheit der story betont, zum anderen jedoch mit metanarrativen und metaleptischen Erzählverfahren der discourse als Erzählakt hervorgehoben und so die Illusion eines Erzählens erzeugt. ${ }^{9}$ Diese sich widersprechenden

8 Vgl. u. a. zuJakob von Gunten und dem Pikaro-Roman Bernhard Malkmus, The German Picaro and Modernity. Between Underdog and Shape-Shifter (New York, London: Continuum, 2011).

9 Darauf hat Ansgar Nünning aufmerksam gemacht, indem er betont, dass Metanarration keineswegs immer eine „Illusionsdurchbrechung“ sein muss, wie es bei Werner Wolf heißt. Vgl. Ansgar Nünning, „Metanarration als Lakune der Erzähltheorie. Definition, Typologie und Grundriss einer Funktionsgeschichte metanarrativer Erzähleräußerungen“, AAA: Arbeiten aus Anglistik und Amerikanistik 26, Nr. 2 (2001): 125-164; Ansgar Nünning, „Mimesis des Erzählens. Prolegomena zu einer Wirkungsästhetik, Typologie und Funktionsgeschichte des Akts des Erzählens und der Metanarration“, in Erzählen und Erzähltheorie im 2o. Jahrhundert. Festschrift für Wilhelm Füger, hg. von Jörg Helbig (Heidelberg: Universitätsverlag C. Winter, 2001), 13-47; Wolf, Ästhetische Illusion und Illusionsdurchbrechung in der Erzählkunst. 
Modelle treten besonders deutlich hervor, wenn es zu einem Konflikt zwischen der Erzählung und dem Erzähler kommt:

Der [Simon] griff, wie er immer abends zu tun pflegte, in die Saiten seiner Mandoline, und die Erzählung streitet mit mir über den Punkt, was süßer gewesen sei, das Spiel der behenden Finger oder die stillen Frauenaugen, welche auf den Spieler herabsahen. (SW 2, 2of.)

Simon wird als Figur im Rahmen des Fiktionalitätspaktes dargestellt und gleichzeitig im metanarrativen Einschub als Fiktion im Rahmen einer Erzählillusion konzeptualisiert. Diese Erzählillusion ihrerseits wird damit fiktionalisiert und wegen der ungewöhnlichen Aufspaltung zwischen Erzähler und der personifizierten Erzählung wenig glaubhaft. Es ist an dieser Stelle auch zu beobachten, wie sich die Erzählung im „Spiel der behenden Finger" Simons spiegelt und die Rezeption in den "stillen Frauenaugen“ aufgefangen wird. Der ,Streit" der Erzählung mit dem Ich-Erzähler - schon dies eine Metalepse -, darüber, was „süsser gewesen sei“, ist derjenige nach der Bedeutung von Produktion und Rezeption, Sprechen und Hören, Schreiben und Lesen und betont das enge und ambivalente Verhältnis. Auf mehrfach hintertriebene Weise wird so auf ein klassisches Kommunikationsmodell referiert, das dazu dient, das Spiel mit der Ordnung zu intensivieren.

Auch auf der Inhaltsseite wird Performanz vielfach herausgestellt. So spielen Simon und die Herrin ihre Rollen im vollen Bewusstsein ihres Spiels: "Sie spielten nun Tag für Tag Herrin und Page, und befanden sich wohl dabei. Simon war es ernst." (SW 2, 20) Identitäten werden performativ über Rollenspiele hergestellt, zeigen sich dadurch in ihrer Kontingenz und Konstruiertheit und nicht als verlässliche Referenzen. Dass dieses Spiel ernst ist, also an

Martin Jürgens, der sich als einer der wenigen dieser frühen Erzählung Walsers angenommen hat, betont nur den Aspekt der Illusionsdurchbrechung: „Die Realität des Beschriebenen wird so durch die Einführung der Erzählung als Figur - mit ihrem Anspruch auf Distanz vom zu Beschreibenden - als Fiktionalität erkennbar gemacht. Es ist nicht die Erzählung, die sich nach ihrem Gegenstand, der Figur Simon richtet, sondern umgekehrt: Der Gegenstand, Simon, erscheint als das Kunstprodukt der Erzählung als einer handelnden Figur." Martin Jürgens, Robert Walser. Die Krise der Darstellbarkeit. Untersuchungen zur Prosa (Kronberg Taunus: Scriptor, 1973), 31. Jürgens betont das Spielerische der früheren und mittleren Prosa Walsers hinsichtlich dessen Thematisierung von der - gemäß Jürgens These - Darstellbarkeit der Welt: „In der Geschichte Simon, wie fast durchweg in der frühen und mittleren Prosa Robert Walsers, ist diese in ihr bereits angelegte Dualität [von Formanspruch der Erzählung und Vermittlung des Erzählers] jedoch noch aufgehoben in der Form einer spielerischen literarischen Reflexion, die im Verlauf des Textes einmal die Determinationskraft des Gegenstandes, einmal die Verfügungsgewalt des sprechenden Ichs über ihn, einmal die Erfordernisse der Erzählung selbst (als Figur) hervorhebt." Jürgens, 32. 
die identitätssetzende Kraft der Performanz geglaubt wird, wird explizit betont. Am Schluss von Simon. Eine Liebesgeschichte wird diese Kraft nachdrücklich sichtbar gemacht: Die Performanz der Erzählung rettet Simon und seine Herrin vor deren früherem Mann, Aggapaia, der vor der Schlosstüre im Nachsinnen über das, was passiert sein könnte, erstarrt:

„Er überlegt“, sang siegesfroh des Pagen frische Stimme. Er überlegt noch heute, der arme, schwarze Teufel Aggapaia. Er klebt an seiner Überlegung fest. Simon und Klara sind Mann und Weib geworden. (SW 2, 22)

Dass die Erzählung die beiden wider alle Wahrscheinlichkeit „Mann und Weib“ werden lässt, muss nicht begründet werden. In einer Referenz auf den plötzlichen Entschluss Simons, „sofort das zu werden, wozu ihn die unbändige Lust anfeuerte" (SW 2, 15), der die Geschichte ins Rollen brachte und den Erzählakt startete, wird die Geschichte ebenso abrupt in metanarrativer Sprechweise beendet: „Wie? sagt später einmal die Geschichte, welche hier atemringend der Ruhe bedarf." (SW 2, 22) Paradoxe Erzählstrukturen, divergierende Poetiken und vieldeutige Begrifflichkeiten sorgen in Simon nicht für eine defizitäre Erzählung, sondern, im Gegenteil, sind konstitutiv für den Text selbst. ${ }^{10}$ Indem die Performanz zur Figur wird, das Rollenspiel inszeniert und doch wirklich ist, Erzähler und Erzählung sich nicht ertragen und doch in denselben Text gehören, erzeugt Simon die narrative Quadratur des Kreises.

\subsubsection{Prekäres Erzählen}

Mit der Lektüre von Simon. Eine Liebesgeschichte sind die narrativen Verfahren skizziert worden, mit denen Robert Walser Ordnungen auf verschiedenen

10 Ähnliches zeigt Reto Sorg für Das „Tagebuch“-Fragment 1926: „Walsers Tagebuch-Erzählung dagegen unterläuft nicht nur die, romantische Fabrikation', sondern ebenso die Trennung von Wirklichkeit und Imagination. So etwas wie,Wirklichkeit‘ und, Identität' erscheint nur mittels eines Erzählens vorstellbar, das Faktuales und Fiktionales nicht trennt, sondern nolens volens vermischt. [...] Die Selbstdarstellung von Walsers Erzähler tendiert weder zur Festschreibung einer eindeutigen Identität, die mit der Präsentation eines ,wahren Ich einherginge, noch zu einem hyper-modernen Maskenspiel, in dem Identität aus Rollen eines unfassbaren Ich besteht. Die Erzählhaltung kultiviert vielmehr die Ambivalenzen und entwickelt aus der ,Zwei- oder Mehrbedeutigkeit' der Dinge ein dynamisches Narrativ, das die Produktivität, aber auch die Brüchigkeit der selbstbewussten modernen Existenz repräsentiert." Reto Sorg, „Jäger und Gejagter in ein und derselben Person. Zur ,Zweibedeutigkeit‘ in Robert Walsers Tagebuch-Erzählung von 1926“, in Robert Walsers Ambivalenzen, hg. von Kurt Lüscher u. a., 2., durchgesehene und korrigierte Aufl., Robert Walser-Studien 3 (Paderborn: Wilhelm Fink, 2019), $126 \mathrm{f}$. 
Ebenen unterläuft und eine neue, prekäre Ordnung etabliert..11 Indem die Erzählung ihren eigenen Erzählprozess darstellt, trifft sie den Kern dessen, was prekäres Erzählen ausmacht. Prekäres Erzählen ist ein Akt, der zwangsläufig seinen Konstruktionscharakter betont und seine Künstlichkeit, deren Ordnung und Ordnungsverfahren nicht notwendig, sondern kontingent sind, herausstellt. In dieser Fokussierung auf den Akt entspricht es eigentümlich präzise der Etymologie von "prekär" als "wünschen, auf Widerruf gewähren“ in der Betonung des Vorläufigen, Nicht-Fixierten und Performativen..$^{12}$ Mehr noch als ein bloßes Zeigen von Kontingenz, Brüchigkeit und Unsicherheit ist es für das prekäre Erzählen, wie es hier etabliert wird, zentral, dass diese Prekarität wiederum konstitutiv für die Erzählung ist. Aufgesetzte Metalepsen oder redundante, unmotivierte Ereignisse, Elemente und Verfahrensweisen, die einem Text beigefügt sind, qualifizieren sich solange nicht als prekäres Erzählen, als diese Verfahren nicht tief in den Strukturen verwurzelt sind und eine Dringlichkeit für die Erzählung selbst haben: Das Prekäre des Erzählens ist konstitutiv für die Erzählung. Dies ist die interpretatorisch zu ziehende

11 Das Adjektiv „prekär“ wird heute in einem breiten Spektrum verwendet. Insbesondere in den Sozialwissenschaften und dem öffentlichen Diskurs zur Beschreibung von unsicheren Arbeitsverhältnissen ist es wichtig. Es ist in diesem Zusammenhang vielfach die Rede von einem ,neuen Prekariat', was sich häufig auf akademische Anstellungsbedingungen, die sogenannte ,Generation Praktikum' oder die Arbeitsverhältnisse von Migrantinnen und Migranten bezieht. Vgl. etwa Claudio Altenhain u. a., Hrsg., Von „Neuer Unterschicht" und Prekariat. Gesellschaftliche Verhältnisse und Kategorien im Umbruch. Kritische Perspektiven auf aktuelle Debatten (Bielefeld: transcript, 2008); Nadine Sander, Das akademische Prekariat. Leben zwischen Frist und Plan (Konstanz: UVK, 2012); Irene Götz und Barbara Lemberger, Hrsg., Prekär arbeiten, prekär leben. Kulturwissenschaftliche Perspektiven auf ein gesellschaftliches Phänomen, Arbeit und Alltag (Frankfurt am Main, New York: Campus, 2009). In diesem Zusammenhang ist die deutlich ansteigende Verwendung des Ausdrucks seit den 195oer-Jahren interessant. Vgl. „Prekär', bereitgestellt durch das Digitale Wörterbuch der deutschen Sprache“, zugegriffen am 2. Mai 2020, https://www.dwds.de/wb/prekär.

In den Literaturwissenschaften wird das Attribut "prekär“ nicht spezifisch verwendet. Wenn, dann wird es im Wortsinn zur Beschreibung gesellschaftlicher Verhältnisse, Figurenkonstellationen und Lebensumstände von Figuren und Autoren eingesetzt. Jüngere Studien untersuchen die prekäre Arbeit in literarischen Darstellungen. Vgl. Liam Connell, Precarious Labour and the Contemporary Novel (New York: Springer, 2017). Dagegen scheint der Begriff in den Theaterwissenschaften relevant zu werden, in denen mit Judith Butler (Precarious Life) und Emmanuel Levinas die ethischen Komponenten von Macht und Körper wichtig werden. So in Mireia Aragay und Martin Middeke, Hrsg., Of Precariousness. Vulnerabilities, Responsibilities, Communities in 21st-Century British Drama and Theatre (Berlin, Boston: De Gruyter, 2017), https://doi.org/10.1515/9783110548716.

12 Vgl. Wolfgang Pfeifer, Hrsg., „Prekär“, in Etymologisches Wörterbuch des Deutschen (1993), digitalisierte und von Wolfgang Pfeifer überarbeitete Version im Digitalen Wörterbuch der deutschen Sprache, zugegriffen am 2. Mai 2020, https://www.dwds.de/wb/prekär. 
Grenze des Begriffs. In dieser Begrenzung setzt der Begriff sich auch deutlich vom Konzept der unnatural narratives ab. ${ }^{13}$

Mit der Schärfung des Blicks auf das Verhältnis vom Erzählen zur Ordnung in ihrer ganzen Komplexität ermöglicht das Konzept des prekären Erzählens fruchtbare Lektüren, Kontextualisierung von Erzählformen und lässt ungewöhnliche Verwandtschaften von Texten deutlich werden. Prekäres Erzählen ist kein rein analytischer Begriff, sondern ein interpretativer, der auf der Analyse von Erzählverfahren, story und symbolischen Ordnungen beruht. ${ }^{14}$ Prekäre Erzählungen hinterfragen auf vielfältige Weise ihre eigenen Ordnungen. Gerade dadurch wird das Prekäre auf eine paradoxe Weise konstitutiv für dieses Erzählen. Nicht selten äußert sich darin eine neue Souveränität des Erzählens, die nicht an herkömmliche Erzählordnungen gebunden zu sein scheint.

In dieser Hinsicht erinnert die Methodik in der Analyse prekären Erzählens an Verfahren der Dekonstruktion. Die Parallele besteht in der Aufmerksamkeit auf Widersprüchliches im Text, auf konkurrierende Bedeutungen, auf paradoxe, vor dem Hintergrund des Erzählmodells als Repräsentation eines

13 Dies gilt insbesondere für die Spielart von unnatural narratives, die Jan Alber vertritt. „[P]hysically, logically, and humanly impossible scenarios and events“ müssen noch kein prekäres Erzählen darstellen, können doch auch konventionell erzählte Sience-FictionRomane in diesem Sinn unnatural sein. Jan Alber, Unnatural Narrative. Impossible Worlds in Fiction and Drama (Lincoln, London: University of Nebraska Press, 2016), 25. In dem hier vorgeschlagenen Verständnis sollte erst dann von prekärem Erzählen gesprochen werden, wenn dadurch die eigene Ordnung als prekäre Ordnung reflektiert wird und dies für die Erzählung zentral ist. Siehe Kap. 5.4 Antinarratives?, S. 263ff. unten.

Analog gilt für Moritz Baßlers „Pinguin-Effekt“, der die Irritation durch ein unstimmiges Detail bezeichnet, „das sich der einfachen hermeneutischen Lesart widersetzt“, dass dieser erst dann prekäres Erzählen ausstellt, wenn er nicht nur aufgesetzt, sondern konstitutiv ist. Vgl. Moritz Baßler, Die Entdeckung der Textur. Unverständlichkeit in der Kurzprosa der emphatischen Moderne 1910-1916 (Tübingen: Max Niemeyer, 1994), 108; zum Pinguin-Effekt vgl. Baßler, 108-113.

14 Damit sei auch darauf hingewiesen, dass in dieser Arbeit nicht davon ausgegangen wird, dass die Narratologie eine rein analytische Wissenschaft zu sein habe, die sich als objektive Wissenschaft von der Interpretation abzugrenzen versucht, wie es im Strukturalismus der 196oer-Jahre propagiert wurde. Schon die Unterscheidung von Sequenzen innerhalb einer Erzählung als Grundlage der Analyse stellt große Herausforderungen dar. Die Unterscheidung von Analyse und Interpretation wird dennoch auch mit einigem Grund aufrechterhalten. Vgl. etwa Jan Christoph Meister, „Narratology as Discipline. A Case for Conceptual Fundamentalism“, in What is Narratology? Questions and Answers Regarding the Status of a Theory, hg. von Tom Kindt und Hans-Harald Müller, Narratologia. Contributions to Narrative Theory 1 (Berlin, Boston: De Gruyter, 2003), 55-71. Vgl. auch Tilmann Köppe und Tom Kindt, Erzähltheorie. Eine Einführung, Universal-Bibliothek (Stuttgart: Reclam, 2014). 
vergangenen (fiktiven) Ereignisses eigentlich unmögliche Erzählsituationen sowie auf alternative Ordnungsformen. Der Fokus des prekären Erzählens liegt jedoch insbesondere auf dem Erzählen selbst, ist gegenüber der Dekonstruktion, die den Fokus auf die Semantik und Semiotik legt, spezifischer und wendet das narratologische Instrumentarium an. Zudem ist es die Absicht des Konzepts des prekären Erzählens, der Tendenz der Dekonstruktion, den Bedeutungsgehalt zugunsten der Betonung der Unmöglichkeit von Bedeutung zu vernachlässigen, zu entgehen und zu untersuchen, wie Ordnung jenseits von konventionellen Erzählordnungen im Erzählen der Moderne hergestellt wird. Das prekäre Erzählen als Kategorie soll also einen Mittelweg einschlagen zwischen der „ambiguitätsversessene[n]“ Dekonstruktion und der „ambiguitätsvergessene[n] " Kulturwissenschaft, die Ordnung tendenziell als kohärentes Sinnsystem beschreibt. ${ }^{15}$ Es dient hier gleichzeitig als methodische Leitlinie, indem Texte auf Ordnung und Ordnungsherstellung untersucht werden, wie als theoretisches Konzept, das durch die Lektüren der Primärtexte differenziert, mit konkreten Erzählverfahren und Techniken angereichert sowie schließlich an zeitgenössische narratologische Konzepte angebunden wird.

\subsubsection{Drei Formen des prekären Erzählens. Walser, Kafka und Fontane}

Die Thematisierung von Ordnung und ihrer Prekarität wird in dieser Studie zum gemeinsamen Grund, auf dem Walser, Kafka und Fontane nebeneinandergestellt werden. So unterschiedlich sich deren jeweilige Positionen zu Ordnung und so gegensätzlich sich ihre Umgangsweisen mit dem Ordnungsproblem darstellen, so deutlich sind alle drei als implizite Theoretiker und explizite Praktiker narrativer Ordnungen zu verstehen. Die drei Autoren, deren hier behandelte Texte in einem Zeitraum von rund 30 Jahren entstanden, werden als Vertreter von drei prototypischen Umgangsweisen mit einer prekär gewordenen Ordnung gelesen. Bei Fontane lässt sich, vereinfacht, der Versuch der Kontrolle des Prekären feststellen, bei Walser eine Befreiung des Narrativen in die Performanz und bei Kafka die Etablierung einer aporetischen Ordnung.

Dass Kafka sich mit Ordnung und deren Herstellung auseinandersetzte, dürfte auf semantischer Ebene am unmittelbarsten deutlich sein. Seine großen, fragmentarischen Romane sind immer auch Auseinandersetzungen mit spezifischen, undurchsichtigen Ordnungen. Ist es in Der Process und Das

15 Julia Abel, Andreas Blödorn und Michael Scheffel, „Narrative Sinnbildung im Spannungsfeld von Ambivalenz und Kohärenz. Einführung“, in Ambivalenz und Kohärenz. Untersuchungen zur narrativen Sinnbildung, hg. von Julia Abel, Andreas Blödorn und Michael Scheffel (Trier: Wissenschaftlicher Verlag Trier, 2009), Iff. Siehe Kap. 1.3.3 Ordnung in Rhetorik und Narratologie, S. zoff. unten. 
Schlo $\beta$ jeweils die Macht selbst, deren Ordnung im Kern unergründlich ist, so zeichnet Der Verschollene eine Ordnung zwischen alter und neuer Welt, zwischen Künstlerdasein und Bürgerlichkeit. Auch in zahlreichen Erzählungen werden Ordnungen und deren Herstellungsverfahren modelliert. Ganz besonders deutlich und geradezu isoliert wird dieses Problem der Ordnung im späten Text Der Bau dargestellt, der hier im Zentrum steht. Ordnung ist bei Kafka auch auf der Ebene der Verfahren von großer Wichtigkeit, sowohl in den vorgeführten Metaphern der Generierung von Bedeutung wie auch in den konkreten Erzählverfahren selbst.

Die Nähe von Walser und Kafka ist vielfach betont worden. ${ }^{16}$ Schon Robert Musil nannte in einer Sammelrezension, in der Walsers Band Geschichten (Kurt Wolff, 1914) und Kafkas erste Buchpublikation Betrachtung (Rowohlt, 1913) besprochen wurden, letzteren einen "Spezialfall des Typus Walser“. ${ }^{17}$ Von Kafka ist überliefert, dass dieser Walser schätzte, insbesondere die Romane Der

16 Vgl. dazu Georg Kurscheidt, „,Stillstehendes Galoppieren` - der Spaziergang bei Robert Walser. Zur Paradoxie einer Bewegung und zum Motiv des ,stehenden Sturmlaufs' bei Franz Kafka“, Euphorion 81 (1987): 131-155; Vesna Kondrič Horvat, Franz Kafka und Robert Walser im Dialog (Berlin: Weidler, 2010); darin besonders Karl Pestalozzi, „Spazieren und Schreiben. Franz Kafka ,Der plötzliche Spaziergang' und Robert Walser ,Der Spaziergang'“, in Franz Kafka und Robert Walser im Dialog, hg. von Vesna Kondric Horvat (Berlin: Weidler Buchverlag, 2010), 23-40; Sabine Rothemann, Spazierengehen Verschollengehen. Zum Problem der Wahrnehmung und der Auslegung bei Robert Walser und Franz Kafka (Marburg: Tectum Verlag, 200o).

17 Robert Musil, „Literarische Chronik“, Die neue Rundschau 7 (Juli 1914): 1169. In Auszügen wieder abgedruckt in Robert Musil, „Die Geschichten von Robert Walser“, in Über Rober Walser, hg. von Katharina Kerr, Bd. 1, 3 Bde. (Frankfurt am Main: Suhrkamp, 1978f.), 89-91. $\mathrm{Zu}$ dieser einflussreichen Rezension Musils vgl. Peter Henninger, „Erlebnis, Dichtung und Kritik in Robert Musils ,Literarischer Chronik‘ vom August 1914“, hg. von Norbert Christian Wolf und Rosmarie Zeller, Musil-Forum 31 (2009/2010): 193-201, https://doi. org/10.1515/9783110271218.193.

Robert Musil ließe sich auch gewinnbringend in die Diskussion um das prekäre Erzählen einbringen. Die Erzählungen in Drei Frauen sind als Reaktion auf das Ordnungsproblem der Moderne zu lesen, die in der Konstruktion einer quasi-mythischen Ordnung besteht, während in den frühen Vereinigungen auch eine sozusagen sprachliche Nachbildung dieser Ordnung stattfindet. Im Mann ohne Eigenschaften, so wäre zu argumentieren, wird das Ordnungsproblem in der extensiven und unabschließbaren Reflexion im Essayistischen sowie der ansatzweisen Nüchternheit der Neuen Sachlichkeit beleuchtet.

Die Relation von Kafka und Walser hat sich heutzutage umgekehrt: Wurde ehedem Kafka mit Walser verglichen, um seine Bedeutung hervorzuheben, so geschieht heute das Gegenteil und Walser wird mit der Wertschätzung, die Kafka ihm entgegenbrachte, angepriesen. Interessant ist, wie Walser um 1913 als Referenz fungierte und bei Reiner Stach gleichzeitig "der noch beinahe unbekannte Robert Walser“ ist. Reiner Stach, Kafka. Die Jahre der Entscheidungen (Frankfurt am Main: Fischer, 2002), 382. 
Gehülfe und Jakob von Gunten. Das Bindeglied zum 1898 verstorbenen Fontane ist die Ordnungsproblematik der Moderne, die insbesondere in Fontanes letztem Roman zum Thema wird. Der Stechlin ist ein Roman des Übergangs. Zeitlich steht er mitten in der Moderne, spielt in Teilen in der Großstadt Berlin und ist geprägt von modernen Diskursen zur Bedeutung der Technik, des Kapitalismus, der Demokratie und der Sozialen Frage. Diese Elemente werden durch das Setting im ländlichen Stechlin, die Figurenzeichnung und insbesondere die Erzählweise harmonisiert. Der gemächliche Ton des Stechlin steht in unübersehbarer Spannung zu dieser Thematik: Es ist der discourse, der die prekäre Ordnung der story, ihre Komplexität und die Ambivalenz der Wertungen zu kontrollieren scheint und so den Eindruck einer gemütlichen Bürgerlichkeit evoziert. ${ }^{18}$

Was sich in Theodor Fontanes Alterswerk Der Stechlin ansatzweise und in engen Grenzen darstellt, die teilweise noch von den Epochengrenzen des Realismus stammen, findet bei Robert Walser und Franz Kafka eine je eigene Form radikaler Entfaltung. Die drei Autoren bilden also verschiedene Umgangsformen mit und Darstellungsweisen von Ordnung und deren Problematik in der Moderne, die zusammen eine adäquate Breite dieser Untersuchung ermöglichen.

\subsection{Moderne als Ordnungsproblem}

Das prekäre Erzählen, als Subversion und Neugestaltung von Erzählordnungen in inhaltlicher wie formaler Hinsicht, hat seinen kulturhistorischen Hintergrund in der Moderne als Ordnungsproblem. Im Folgenden wird die Basis geschaffen für das Verständnis der Ordnungsleistungen Walsers, Kafkas und Fontanes. Zunächst wird mit Zygmunt Bauman die Relation von Ordnung und Ambivalenz als allgemeines Prinzip der Moderne herausgearbeitet. Anschließend wird der vieldeutige Begriff der Moderne in einem weiteren

18 Es wird in dieser Arbeit durchgehend die angelsächsische Dichotomie von story und discourse statt der französischen histoire und discours (beziehungsweis histoire, récit und narration) von Gérard Genette verwendet. Vgl. Seymour Chatman, Story and Discourse. Narrative Structure in Fiction and Film (Ithaca, London: Cornell University Press, 1978); Gérard Genette, Die Erzählung, übers. von Andreas Knop, 3., durchges. und korrigierte Aufl., UTB (Paderborn: Wilhelm Fink, 2010). Vgl. für einen Überblick über weitere Konzepte, die alle grundsätzlich auf derselben Unterscheidung von Wie und Was beruhen: Michael Scheffel, „Narrative Constitution“, in The Living Handbook of Narratology, hg. von Peter Hühn u. a. (Hamburg: Hamburg University, 2013), http://www.lhn.unihamburg.de/article/narrative-constitution; Matias Martinez und Michael Scheffel, Einführung in die Erzähltheorie (München: C. H. Beck, 2007), 20-26; Wolf Schmid, Elemente der Narratologie (Berlin, Boston: De Gruyter, 2014), 230-284. 
Sinn als historische Epoche und in einem engeren als literarische skizziert. Mit Georg Simmel wird sodann auf die ersten zwei Jahrzehnte des 2o. Jahrhunderts fokussiert. In der Folge wird der Begriff der Ordnung selbst erläutert. Hierbei steht Fritz Mauthners Sprachkritik im Mittelpunkt. Schließlich wird die Bedeutung des Ordnungsbegriffs für das Erzählen und die Wissenschaft des Erzählens, die Narratologie, thematisiert sowie der hier verwendete Ambivalenzbegriff skizziert. ${ }^{19}$

\subsubsection{Ordnung und Ambivalenz (Bauman)}

Wir können uns die Moderne als eine Zeit denken, da Ordnung - der Welt, des menschlichen Ursprungs, des menschlichen Selbst, und der Verbindung aller drei - reflektiert wird; ein Gegenstand des Nachdenkens, des Interesses, einer Praxis, die sich ihrer selbst bewußt ist, bewußt, eine bewußte Praxis zu sein und auf der Hut vor der Leere, die sie zurücklassen würde, wenn sie innehalten oder auch nur nachlassen würde. ${ }^{20}$

Zygmunt Bauman lässt die Moderne da beginnen, wo diese ihre eigene Ordnung zu thematisieren beginnt. ${ }^{21}$ Sobald Ordnung reflektiert wird, wird sie nicht mehr als gegeben betrachtet, sondern als etwas, das hergestellt werden

19 Was hier nicht geleistet werden kann, ist eine erschöpfende Aufarbeitung des Begriffs der Ordnung in der Moderne. Zu vielfältig sind seine Verwendungsweisen, zu lang ist seine Geschichte. Statt einer diskursanalytischen Aufarbeitung der Verwendungsweise des Begriffs Ordnung im behandelten Zeitraum in zeitgenössischen Zeitschriften und Zeitungen werden mit Simmel und Mauthner einschlägige Referenzpositionen verhandelt. Für einen allgemeinen Überblick vgl. Helmut Meinhardt u. a., „Ordnung“, in Historisches Wörterbuch der Philosophie, hg. von Joachim Ritter, Karlfried Gründer, und Gottfried Gabriel, Bd. 6, 13 Bde. (Basel: Schwabe, 2017), 1249-1309, https://doi.org/10.24894/HWPh.5661; für einen Fokus auf die Politik- und Wirtschaftswissenschaften: Andreas Anter, Die Macht der Ordnung. Aspekte einer Grundkategorie des Politischen (Tübingen: Mohr Siebeck, 2004); für die Soziologie: Oskar Negt, Die Konstituierung der Soziologie als Ordnungswissenschaft. Strukturbeziehungen zwischen den Gesellschaftslehren Comtes und Hegels, 2. Aufl., Studien zur Gesellschaftstheorie (Frankfurt: Europäische Verlagsanstalt, 1974); für den Kontext in der Philosophie: Helmut Kuhn und Franz Wiedmann, Hrsg., Das Problem der Ordnung (Meisenheim am Glan: Verlag Anton Hain, 1962).

20 Zygmunt Bauman, Moderne und Ambivalenz. Das Ende der Eindeutigkeit, übers. von Martin Suhr (Hamburg: Junius, 1992), 17. Hervorhebung i. O.

21 Hans Blumenberg sieht die Neuzeit aufs Engste verknüpft mit einem „Ordnungsschwund“: „Unter dem Namen des ,Ordnungsschwundes“ suche ich die epochale Krise zu erfassen, die das geistige Gepräge der Neuzeit bestimmt hat." Hans Blumenberg, „Ordnungsschwund und Selbstbehauptung. Über Weltverstehen und Weltverhalten im Werden der technischen Epoche“, in Das Problem der Ordnung, hg. von Helmut Kuhn und Franz Wiedmann (Meisenheim am Glan: Verlag Anton Hain, 1962), 40. Bei Blumenberg wird dieses „Unordnungsaxiom“ in ein technisiertes Ordnungsprinzip umgekehrt. Blumenberg, 56 . 
muss. Diesen Wandel des Verständnisses von Ordnung als etwas Gegebenes hin zu der Vorstellung, Ordnung sei etwas zu Konstruierendes, also den Wandel vom Natürlichen zum Künstlichen, versteht Bauman als eigentlichen Beginn der Moderne.

Den historischen Beginn der Moderne zu bestimmen, ist nach Bauman schwierig, da die Festlegung des Beginns selbst schon Teil der Moderne ist. Der Einfachheit halber setzt Bauman die Problematisierung von Ordnung - in Dissens mit der konventionellen Epochengrenze vom Mittelalter zur Neuzeit um 1500 - bei Thomas Hobbes und dessen Leviathan (1651) an. Im Leviathan wird die Notwendigkeit einer unbedingten Ordnung postuliert, die den Naturzustand als ordnungsloses Chaos beendet. Hobbes' hegemoniales Staatsgebilde fußt auf einfachen Regeln, die vom postulierten Naturrecht abgeleitet werden können und sich durch dieses rechtfertigen. Damit ist auch die Zuschreibung von Ordnung und Chaos an eine Perspektive gebunden. $\mathrm{Zu}$ bestimmen, was Ordnung und was Chaos ist, ist eine Funktion der Macht und geschieht naturgemäß aus einer Position der Ordnung heraus. ${ }^{22}$

Bauman beschreibt den Prozess der fortwährenden Ausdifferenzierung der Ordnung als Resultat von wachsenden Erkenntnisinteressen. Die Differenzierung als Erzeugen immer feinerer Unterscheidungen kann mit Bauman als die grundlegende Operation von Ordnung verstanden werden. Ordnung wendet sich dabei gegen die Ambivalenz als das Andere der Ordnung. Diesen „Kampf“ um die Auslöschung der Ambivalenz beschreibt Bauman als „typisch moderne Praxis“. ${ }^{23}$

Der Kampf um Ordnung ist nicht ein Kampf der einen Definition gegen eine andere, einer Möglichkeit, Realität auszudrücken, gegen eine andere. Es ist ein Kampf der Bestimmung gegen die Mehrdeutigkeit, der semantischen Präzision gegen Ambivalenz, der Durchsichtigkeit gegen Dunkelheit, der Klarheit gegen Verschwommenheit. [...] Das andere der Ordnung ist nicht eine andere Ordnung: Die einzige Alternative ist das Chaos. ${ }^{24}$

22 Vgl. dazu Andreas Anter in seiner Habilitationsschrift, die den für die Sozialwissenschaften zentralen Ordnungsbegriff systematisch bearbeitet: „Denn jede Ordnungsbildung bleibt natürlich immer ein Herrschaftsproblem.“ Anter, Die Macht der Ordnung, 3o. Dabei gilt auch: „Jede Ordnung ist zugleich eine Machtordnung." Anter, 93.

23 "Die typisch moderne Praxis, die Substanz moderner Politik, des modernen Intellekts, des modernen Lebens, ist die Anstrengung, Ambivalenz auszulöschen: eine Anstrengung, genau zu definieren - und alles zu unterdrücken oder zu eliminieren, was nicht genau definiert werden konnte oder wollte." Bauman, Moderne und Ambivalenz, $20 f$. 
Jedoch entsteht da, wo eine Unterscheidung gemacht wird, zugleich die Möglichkeit von Ambivalenz. Mit der Potenzierung der Ordnung potenziert sich die Ambivalenz. So wird der Kampf gegen die Ambivalenz zum Erzeuger von Ambivalenz. In diesem Sinn ist dieser Kampf „selbstmörderisch und selbsterzeugend“. ${ }^{25}$ Ordnung und Chaos begreift Bauman als „moderne Zwillinge“. ${ }^{26}$

Mit dieser engen Bindung wird Ordnen zu einem selbstreferenziellen Prozess. Was geordnet wird, muss zunächst erzeugt werden: Die Ordnung konstruiert im Prozess des Ordnens, was sie ordnet. Das heißt auch, dass das Herstellen von Ordnung die Prekarisierung von Ordnung mit sich führt. Jede neue Differenz als Basisoperation des Ordnens erzeugt eine neue Ambivalenz zwischen den zwei Seiten der Unterscheidung. ${ }^{27}$ Diese Denkfigur ermöglicht es, Ordnung als etwas Prekäres zu verstehen, das sein Gegenteil mitträgt und gleichzeitig auf diesen Antagonismus angewiesen ist.

Chaos, „das Andere der Ordnung“, ist reine Negativität. Es ist die Verneinung all dessen, was Ordnung zu sein sucht. Gegen eben diese Negativität konstituiert sich die Positivität der Ordnung. Aber die Negativität des Chaos ist ein Produkt der Selbstkonstitution der Ordnung: ihre Nebenwirkung, ihr Abfall, und gleichwohl die conditio sine qua non ihrer (reflexiven) Möglichkeit. Ohne die Negativität des Chaos gibt es keine Positivität der Ordnung; ohne Chaos keine Ordnung. ${ }^{28}$

\subsubsection{Zum Begriff der Moderne}

In der Begriffsgeschichte der Moderne findet sich, was Bauman als Dynamik zwischen Ordnung und Ambivalenz beschreibt. Nicht nur haben sich in der Begriffsgeschichte die Bedeutungen von modern und dessen Gegenteilen, wie alt oder konservativ, radikal geändert; ${ }^{29}$ das Moderne als Begriff ist gleichsam auf Spannung angewiesen:

25 Bauman, 16.

26 „Ordnung ist, was nicht Chaos ist; Chaos ist, was nicht ordentlich ist: Ordnung und Chaos sind moderne Zwillinge." Bauman, 17. Hervorhebung i. O.

27 Vgl. dazu Anter: „Ordnungsbehauptungen bleiben immer prekär“. Anter, Die Macht der Ordnung, 1.

28 Bauman, Moderne und Ambivalenz, 19 f. Hervorhebung i. O.

29 So in der Querelle des Anciens et des Modernes, was „immer wieder zu der paradoxen oder auch dialektischen Situation [führte], daß die fortgeschritteneren und bedeutenderen Denker, Künstler und Kritiker es mit dem ,antico' oder mit den ,anciens‘ gegen das zunächst nur zeitlich jüngere oder nur eben in Mode gewesene Moderne hielten." Rainer Piepmeier, „Modern, die Moderne“, in Historisches Wörterbuch der Philosophie, hg. von Joachim Ritter, Karlfried Gründer und Gottfried Gabriel, Bd. 6 (Basel: Schwabe, 2017), 54, https://www.doi.org/10.24894/HWPh.2571. 
Die Vorstellung des Modernen, Gegenwärtigen, Neuen steht nie für sich allein, sondern fordert immer den Vergleich, die Entgegensetzung, die „Kontrastspannung" heraus: entweder zu einem Alten und Vergangenem oder zu einer Zukunft, einem Morgen, einem Neubeginn, an dessen Vorabend oder Anfang die Moderne sich sieht. ${ }^{30}$

Damit einher gehen die kontroversen Bewertungen, welche die jeweiligen Bewegungen der Moderne erhalten und auch gegenüber anderen Ansichten vertreten. Die ambivalente Bewertung, die Polemik der Diskursteilnehmer, das „Schwanken zwischen Verdammung und Verherrlichung, die Spannung zwischen Fortschritt und Dekadenz, zwischen Herkunft und Zukunft, Nostalgie und Utopie“ ist ein Grundmerkmal des Sprechens über das Moderne während Jahrhunderten. ${ }^{31}$

In der Begriffsgeschichte der Moderne lassen sich mehrere Wendepunkte festmachen. ${ }^{32}$ So ist in der Zeit um 1800 „die für die Begriffsgeschichte von modern/Moderne entscheidende Schwelle [zu] sehen“. ${ }^{33}$ Der Grund dafür liegt in der „Zeit- und Geschichtserfahrung der gebildeten und gelehrten Zeitgenossen selbst. [...] Etwa um 1800 ist die ,Wasserscheide‘ erreicht, an welcher sich die Orientierung der Gegenwart von Herkunft auf Zukunft umstellt.“34 Erst mit Friedrich Schiller und Friedrich Schlegel findet nach Cornelia Klinger - in Dissens zu Zygmunt Bauman - begriffsgeschichtlich ein „erstes Selbstreflexivwerden der Moderne“ ${ }^{\text {35 }}$ statt:

Aufgrund der revolutionären Umbruchsituation hoffen Schiller und seine Zeitgenossen auf ein bald bevorstehendes Ende ihres durch Ausdifferenzierung, Mechanisierung und Arbeitsteilung charakterisierten Zeitalters. [...] Jedenfalls erwarten sie den Anbruch einer ganz anderen Moderne $[\ldots] .^{36}$

30 Cornelia Klinger, „Modern/Moderne/Modernismus“, in Ästhetische Grundbegriffe. Historisches Wörterbuch in sieben Bänden. Studienausgabe, hg. von Karlheinz Barck u. a., Bd. 4 (Stuttgart, Weimar: Metzler, 2002), 122.

31 Klinger, 123.

32 Für eine ausführliche Aufarbeitung des Begriffs seit den frühsten Verwendungsweisen um 5 oo sowie ausführliche weiterführende Literaturangaben vgl. Hans Ulrich Gumbrecht, "Modern“, in Geschichtliche Grundbegriffe. Historisches Lexikon zur politisch-sozialen Sprache in Deutschland. Studienausgabe, hg. von Otto Brunner, Werner Conze und Reinhart Koselleck, Bd. 4, 8 Bde. (Stuttgart: Klett-Cotta, 2004), 93-132.

33 Klinger, „Modern/Moderne/Modernismus“, 129.

34 Klinger, 129.

35 Klinger, 131.

$36 \quad$ Klinger, 132 . 
Mit den revolutionären Bewegungen des Vormärz und des Jungen Deutschland um 1830 ging dann die Überzeugung einher, dass sich die „moderne Schreibart" gerade mit der eigenen Zeit auseinandersetzen muss und sich durch diese definiert. Es ist eine „entschlossene[] Zuwendung zur Gegenwart", die jedoch im „Bewußtsein der Modernität nicht bei allen Vertretern des Jungen Deutschland" gleich vorhanden war. ${ }^{37}$ Die Zuwendung zur Gegenwart brachte auch die Verknüpfung von Leben und Schreiben mit sich, die im politischrevolutionären Engagement gegen die Monarchie wichtig wurde.

Nach 1848 geriet der Modernitätsbegriff des Jungen Deutschland in die Kritik:

Die literarischen Autoren einer neuen Generation nannten sich zwar selbst häufig ,modern', verbanden aber mit dieser Wortverwendung [...] keinerlei programmatischen Anspruch mehr, sondern suchten in bewußter Abwendung vom Aktualitätsstreben ihrer Vorgänger nach überzeitlich gültigen Leitwerten. ${ }^{38}$

Mit dem Aufkommen des Realismus wurde eine eher bewahrende und restaurative Haltung eingenommen. In der zweiten Hälfte des 19. Jahrhunderts kam es zu polemischen Verunglimpfungen des Moderne-Begriffs, der diesen mit dauerhaften negativen Konnotationen belud. ${ }^{39}$ Gleichwohl verstand sich auch der aufkommende Realismus als „modern“. In Theodor Fontanes Aufsatz Unsere lyrische und epische Poesie seit 1848 wird der Realismus als das, was „unsere Zeit nach allen Seiten hin charakterisiert" bestimmt. ${ }^{40}$ Der Realismus sei eine "neue[] Kunstrichtung", eine "moderne Kunst" und so kann Fontane auch vom "Realismus [...] unserer modernen Literatur" sprechen. ${ }^{41}$

Um die Jahrhundertwende ist ein "radikale[r] Wandel[] im Zeiterleben“ festzustellen, der darin mündete, dass die Gegenwart „als nach vorne offener Raum der Handlungsplanung, die sich in Programmen formulieren ließ ${ }^{\prime},{ }^{4}$ verstanden werden konnte. Wenn auch eine lange Entwicklung und $\mathrm{Zu}$ spitzung des Prozesses der Moderne zu beobachten ist, so erfüllt „erst das 20. Jh. [...] den Begriff des modernen Zeitalters in vollem Umfang [...]. [G] esellschaftlich-politische und technologisch-ökonomische Innovationen

\footnotetext{
37 Gumbrecht, „Modern“, 112.

38 Gumbrecht, 115 .

39 Gumbrecht, 114-118.

40 Theodor Fontane, „Unsere lyrische und epische Poesie seit 1848“, in Theodor Fontane. Sämtliche Werke, hg. von Walter Keitel, Bd. 1: Aufsätze. Kritiken. Erinnerungen, Aufsätze und Aufzeichnungen, hg. von Jürgen Kolbe (München: Hanser, 1969), 236.

41 Fontane, 237.

42 Gumbrecht, „Modern“, 120.
} 
[treffen] aufeinander, welche in jeder Hinsicht die Bezeichnung Revolution verdienen. ${ }^{43}$

Kurz vor dieser Hochphase der Moderne wird die deutschsprachige Substantivierung von modern, die Moderne, von Eugen Wolff 1886 bei einem Referat vor der Literarischen Vereinigung Durch! unter dem Titel Die Moderne. Zur "Revolution" und "Reform" der Litteratur geprägt. ${ }^{44}$ Zum ersten Mal geraten diese Begrifflichkeiten ins Zentrum der Aufmerksamkeit. Dabei ist zu beobachten, dass der Diskurs um die Moderne gerade dann anschwillt, wenn der Gegenstand selbst ambivalent wird. So ist der Diskurs um die Moderne zugleich Ausdruck und Versuch der Bewältigung der im Umbruch befindlichen Ordnungsverhältnisse:

Die Hochzeiten des Diskurses um Moderne sind jene, in denen sie prekär wird; die Perspektive, aus welcher der Diskurs hauptsächlich geführt wird, ist eher die der Reflexion, der Skepsis, der Kritik, der Blick von der Peripherie. ${ }^{45}$

Für die literarische Moderne als ästhetisches Phänomen ist die „Komplexität und Ambivalenz des Begriffs und Phänomens Moderne“ zu betonen. ${ }^{46}$ Die literarische Moderne in ihrer Historizität war keineswegs ein einheitliches Projekt. ${ }^{47}$ Gegensätzliche Strömungen innerhalb der Moderne, wie auch antimoderne Bewegungen datieren aus derselben Zeit. ${ }^{48}$ Gerade in der Vielzahl

43 Klinger, „Modern/Moderne/Modernismus“, 138.

44 Eugen Wolff, „Die Moderne. Zur ,Revolution' und „Reform‘ der Litteratur“, Deutsche academische Zeitschrift (Organ der „Deutschen academischen Vereinigung") Jg. 3, Nr. 33 (26. September 1886): Erstes Beiblatt, S. 4 und Zweites Beiblatt, S. 1-2. Online unter: Eugen Wolff, „1886 Eugen Wolff: Die Moderne“, Universität Duisburg-Essen, zugegriffen am 2. Mai 2020, https://www.uni-due.de/lyriktheorie/texte/1886_wolff.html.

Vgl. dazu auch Eugen Wolff, „Die jüngste deutsche Literaturströmung und das Prinzip der Moderne", in Die literarische Moderne. Dokumente zum Selbstverständnis der Literatur um die Jahrhundertwende, hg. von Gotthart Wunberg und Stefan Dietrich, 2., verb. u. komm. Aufl. (Freiburg im Breisgau: Rombach, 1998), 27-82.

45 Klinger, „Modern/Moderne/Modernismus“, 123.

46 Helmuth Kiesel und Sabine Becker, „Literarische Moderne. Begriff und Phänomen“, in Literarische Moderne, hg. von Helmuth Kiesel und Sabine Becker (Berlin, Boston: De Gruyter, 2007), 9. Vgl. dazu auch: Helmuth Kiesel, Geschichte der literarischen Moderne. Sprache, Ästhetik, Dichtung im zwanzigsten Jahrhundert (München: C. H. Beck, 2004).

47 Zeugnis der Vielfalt und Breite des modernen literarischen Schaffens legt auf unterhaltsame Art auch die umfangreiche Anthologie Moritz Baßlers ab: Moritz Baßler, Hrsg., Literarische Moderne. Das große Lesebuch (Frankfurt am Main: Fischer, 2010).

48 Vgl. dazu: „Die Literatur der Moderne changiert [...] im Spannungsfeld einer provokativen Ästhetik oder ästhetischer Provokation auf der einen und literarischer Konvention auf der anderen Seite; sie oszilliert zwischen Literaturrevolution und Tradition, zwischen den Komponenten einer provokativ-experimentellen Moderne und den institutionalisierten 
neuer Bewegungen und Strömungen zeigt sich die Moderne als eine Ausdifferenzierung und damit auch als Versuch des Ordnens. Zeichen davon sind nicht zuletzt die zahlreichen Manifeste, die mit dem naturalistischen Manifest der Vereinigung Durch! bis zu denjenigen der Surrealisten eine eigenständige literarische Gattung stellen. ${ }^{49}$ In ihrem Bestreben nach Abgrenzung sind sie paradoxerweise ihrerseits kontingent. Die Vielzahl der entstehenden Ismen und die zahlreichen Manifeste, die sich durch das prinzipielle Bestreben nach Identität auszeichnen, zeugen so auch von einer Ambivalenz in der Festsetzung dieser Identitäten. Diese Thematisierung von Ordnung in der Moderne als produktive Reaktion spiegelt sich in der Produktivität der Sprachkrise für die Literatur. ${ }^{50}$

\subsubsection{Dynamisierung der Ordnung (Simmel)}

Um die Jahrhundertwende erreichen die tiefgreifenden Veränderungen in den Wissenschaften, der Entwicklung der Städte und vielen weiteren Sozial- und Wissensordnungen einen Höhepunkt. Das Ordnungsproblem der Moderne spitzt sich zu. Von den radikalen Umwandlungen zeugen vielfältige Schlagworte: Verwissenschaftlichung, Industrialisierung, Verstädterung. Insbesondere spielt das Aufkommen der Soziologie als „Ordnungswissenschaft“51 sowohl in der Beschreibung als auch als Ausdruck der Dynamik von Ordnung eine wichtige Rolle.

Georg Simmel hat im Aufsatz Die Großstädte und das Geistesleben (1903) die „tiefsten Probleme des modernen Lebens" auf den Konflikt zwischen Individuum und Gesellschaft zurückgeführt, nämlich dem „Anspruch des Individuums, die Selbstständigkeit und Eigenart seines Daseins gegen die

Ritualen einer traditionelleren und klassisch wirkenden oder zumindest als ,klassisch bezeichneten Moderne.“ Kiesel und Becker, „Literarische Moderne. Begriff und Phänomen“, 10.

49 Vgl. dazu die Sammlung Gotthart Wunberg, Hrsg., Die literarische Moderne. Dokumente zum Selbstverständnis der Literatur um die Jahrhundertwende, 2. Aufl., Rombach Wissenschaft Reihe Litterae (Freiburg im Breisgau: Rombach, 1998).

50 Diesen Aspekt betont Karpenstein-Eßbach: „Dieses Problem der Sprache ist immer wieder als Ausdruck einer Sprachkrise, eines Sprachverlusts oder eines Redeverbots interpretiert worden. Tatsächlich handelt es sich weniger um eine Krise der Sprache, sondern vielmehr um einen Aufstieg der Sprache zum prominenten Thema, das sowohl die Literatur wie die philosophischen, theoretischen und wissenschaftsspezifischen Aussagen der Zeit durchzieht und das für die Verfassung des 2o. Jahrhunderts eine bestimmende Komponente bleiben wird. Die Sorge um die Sprache ist nicht als deren Krise zu verstehen, sondern als ihr Aufstieg zu einer neuen Relevanz." Christa Karpenstein-Eßbach, Deutsche Literaturgeschichte des 20. Jahrhunderts (München: Wilhelm Fink, 2013), 18f. Hervorhebung i. O. Vgl. Negt, Die Konstituierung der Soziologie als Ordnungswissenschaft. 
Übermächte der Gesellschaft, des geschichtlich Ererbten, der äußerlichen Kultur und Technik des Lebens zu bewahren" (GG 116) $)^{52}$. Für Simmel ist dieser Konflikt ein „Grundmotiv“, welches sich seit dem 18. Jahrhundert beobachten lässt und das im „Widerstand des Subjekts, in einem gesellschaftlichtechnischen Mechanismus nivelliert und verbraucht zu werden" (GG 116), besteht.

Dieser Konflikt lässt sich da, wo Simmel das moderne Leben ausmacht, am besten beobachten: in der Großstadt. Bestimmend für das großstädtische Individuum ist nach Simmel die "Steigerung des Nervenlebens" (GG 116, Hervorhebung i. O.), die aus der Vielzahl sich rasch ändernder, disparater und disjunkter Eindrücke, die „rasche Zusammendrängung wechselnder Bilder“ (GG 117) entsteht. Stehen beim Kleinstädter, der in Simmels Argumentation als Gegenpart zur Großstadt wichtig ist, das „Gemüt und gefühlsmäßige Beziehungen“ im Vordergrund, so muss der Großstädter ein „Schutzorgan“ entwickeln "gegen die Entwurzelung, mit der die Strömungen und Diskrepanzen seines äußeren Milieus ihn bedrohen: statt mit dem Gemüte reagiert er auf diese im wesentlichen mit dem Verstande“, dem „von den Tiefen der Persönlichkeit am weitesten abstehende[n] psychische[n] Organ" (GG 117). Diese „Verstandesmäßigkeit“ wird gemäß Simmel zum „Präservativ des subjektiven Lebens gegen die Vergewaltigungen der Großstadt" (GG 118).

Die moderne Verstandestätigkeit stellt Simmel in engen Zusammenhang mit der zunehmenden Bedeutung der Geldwirtschaft, die sich durch reine „Sachlichkeit" auszeichnet (GG 118). Der Handel mit Geld, der alles auf den bloßen Tauschwert reduziert und nivelliert, verunmöglicht einerseits Individualität, da die Handels- und Warenbeziehungen gänzlich anonym bleiben. Diese Wechselwirkung zwischen der Geldwirtschaft und der „intellektualistische[n] Verfassung" ist so eng, dass Ursache und Wirkung nicht bestimmt werden können. „Der moderne Geist ist mehr und mehr ein rechnender geworden.“ (GG 119) Daraus leitet Simmel einen Zwang zu Präzision, Pünktlichkeit und Verbindlichkeit ab, ohne welche die komplexen Beziehungen zwischen den Akteuren nicht mehr funktionieren könnten. Dies verlangt wiederum ein

$5^{2}$ Hier und im Folgenden verweist die Sigle GG auf: Georg Simmel, „Die Großstädte und das Geistesleben [1903]“, in Georg Simmel. Gesamtausgabe in 24 Bänden, hg. von Otthein Rammstedt, Bd. 7.1: Aufsätze und Abhandlungen 1901-1908, hg. von Rüdiger Kramme, Angela Rammstedt und Otthein Rammstedt, 24 Bde. (Frankfurt am Main: Suhrkamp, 1995), 116-131. Der Aufsatz geht zurück auf einen Vortrag, den Simmel im Rahmen eines Vortragszyklus der Gehe-Stiftung im Winter 1902 und 1903 in Dresden gehalten hat. Erstveröffentlicht wurde er 1903 als Georg Simmel, „Die Großstädte und das Geistesleben“, in Die Großstadt. Vorträge und Aufsätze zur Städteausstellung. Jahrbuch der Gehe-Stiftung zu Dresden, hg. von Th. Petermann, Bd. 9 (Dresden, 1903), 185-206. 
„festes, übersubjektives Zeitschema“ (GG 120) und fördert den „Ausschluß jener irrationalen, instinktiven, souveränen Wesenszüge und Impulse [...], die von sich aus die Lebensform bestimmen wollen“ (GG 120).

Doch diese „höchste[] Unpersönlichkeit“ bewirkt andererseits auch ein „höchst [P]ersönliches“, und zwar die „seelische Erscheinung [der] Blasiertheit“, „die Abstumpfung gegen die Unterschiede der Dinge“ (GG 121), die wiederum eine „Entwertung“ der „objektive[n] Welt" wie der „eigene[n] Persönlichkeit" nach sich zieht (GG 122). Aus dieser Abstumpfung resultiert eine „Reserviertheit mit dem Oberton versteckter Aversion“ (GG 123) gegen die Mitmenschen.

Soweit scheint die Großstadt also dem „Unterschiedswesen“ (GG 116) Mensch, der gemäß Simmel fundamental nach Unterscheidung strebt, nicht entsprechen zu können. Was die Großstadt auf dem Weg der „soziale[n] Evolution“ (GG 124) der Kleinstadt und früheren, kleinräumigeren Gesellschaftsformen jedoch voraus hat, ist die persönliche Freiheit: Das Individuum gewinnt „Bewegungsfreiheit [...] und eine Eigenart und Besonderheit, zu der die Arbeitsteilung in der größer gewordenen Gruppe Gelegenheit und Nötigung giebt." (GG 124) Der Mensch wird zur Spezialisierung gezwungen, gerade weil „das Stadtleben den Kampf für den Nahrungserwerb mit der Natur in einen Kampf um den Menschen verwandelt hat" (GG 128). Die Bewohner der Großstadt stehen also nicht mehr in Konkurrenz mit der Natur, sondern mit den Mitmenschen. Dies zwingt zu Spezialisierung und Differenzierung, die zu einer "geistigen Individualisierung seelischer Eigenschaften“ (GG 128) führen. Den Hauptgrund dafür sieht Simmel im „Übergewicht“ des „objektiven Geist[es]“ in der "modernen Kultur“ (GG 129). Mit dem „objektiven Geist" oder „unpersönlich gewordenen Geist[]“ (GG 130) meint Simmel die geistigen Errungenschaften und Institutionen, die eine Tendenz zur Versachlichung aufweisen. In dieser „Hypertrophie der objektiven Kultur" gegen die „Atrophie der individuellen“ muss das Individuum „ein Äußerstes an Eigenart und Besonderung aufbieten [...]; es muß dieses [Persönlichste] übertreiben, um nur überhaupt noch hörbar, auch für sich selbst, zu werden." (GG 130)

Simmel unterscheidet hier zwei Formen der Individualisierung in der Moderne: zum einen diejenige der Freiheit, welche die Großstadt durch ihre Anonymität aufgrund der Größe und den durch die Geldwirtschaft entfremdeten Beziehungen ermöglicht, und zum anderen diejenige der Spezialisierung im Konkurrenzkampf. In diesem Wechselspiel, den Varianten und verschiedenen Möglichkeiten wird die Komplexität und Dynamik in Simmels Beschreibung der modernen Gesellschaft einsichtig.

Die Grundlagen für die Analyse der Großstädte hat Simmel in seinem früheren Werk Über sociale Differenzierung (1890) erarbeitet. Darin betont er 
die extreme Komplexität der modernen Gesellschaft, die Monokausalität ausschließt; implizit wird dadurch die Möglichkeit von Kausalität als globaler Erklärungsstruktur hinterfragt.

Jeder gesellschaftliche Vorgang oder Zustand, den wir uns zum Objekt machen, ist die Erscheinung, bzw. Wirkung unzählig vieler tiefer gelegenen Teilvorgänge. Da nun die gleiche Wirkung von sehr verschiedenen Ursachen ausgehen kann, so ist es möglich, daß die genau gleiche Erscheinung durch ganz verschiedene Komplexe von Kräften hervorgebracht werde, die, nachdem sie an einem Punkte zu der gleichen Wirkung zusammengegangen sind, in ihrer weiteren, darüber hinausgehenden Entwicklung wieder völlig verschiedene Formen annehmen. ${ }^{53}$

Simmel macht hier deutlich, dass Kausalitäten in sich vielfältig sein können und die Beziehung zwischen Ursache und Wirkung keineswegs eindeutig sein muss. Den Hintergrund dieser Komplizierung und Verunsicherung des Prinzips von Ursache und Wirkung bildet die gesellschaftliche Dynamisierung. Gemäß Simmel ist es eine Tatsache, „daß Alles mit Allem in irgend einer Wechselwirkung steht, daß zwischen jedem Punkte der Welt und jedem andern Kräfte und hin- und hergehende Beziehungen bestehen“. ${ }^{54}$ Die zunehmende Komplexität der Gesellschaft und ihrer Analyse zeigt Simmel mit einem grundlegenden Konzept ebendieser Wechselwirkung oder Vergesellschaftung. Bei Simmel bilden die wechselseitigen Beziehungen zwischen den Individuen, und nicht diese selbst, die Basis von Gesellschaft. Wenn er nun weder das Individuum, noch, wie Émile Durkheim, die soziale Tatsache oder, wie Gabriel Tarde, das Gesetz der Nachahmung als primären Pfeiler der sozialen Ordnung postuliert, sondern die Wechselwirkung zwischen Individuen und Institutionen, so betont dies den relationalen und relativen Aspekt von Identität.

Die Dynamisierung von Gesellschaft, Identität und Ordnung bindet Simmel an die historische Moderne:

Die Auflösung der Gesellschaftsseele in die Summe der Wechselwirkungen ihrer Teilhaber liegt in der Richtung des modernen Geisteslebens überhaupt: das Feste, sich selbst Gleiche, Substantielle in Funktion, Kraft, Bewegung aufzulösen und in allem Sein den historischen Prozeß seines Werdens zu erkennen. ${ }^{55}$

53 Georg Simmel, „Über sociale Differenzierung“, in Georg Simmel. Gesamtausgabe in 24 Bänden, hg. von Otthein Rammstedt, Bd. 2: Aufsätze 1887 bis 189o. Über sociale Differenzierung (189o). Die Probleme der Geschichtsphilosophie (1892), hg. von HeinzJürgen Dahme, 24 Bde. (Frankfurt am Main: Suhrkamp, 20o1), $123 f$.

54 Simmel, 130.

55 Simmel, 130. 
Daraus ergibt sich ein dynamisiertes Verständnis von Ordnung: Ordnung ist nicht länger etwas Statisches und Fixiertes, sondern wird zu einem Prozess. ${ }^{56}$ Wie in der Diskussion von Die Großstädte und das Geistesleben deutlich wurde, ist dieser Prozess der Moderne vielgestaltig und umkämpft. Wird Ordnung nur noch über Wechselwirkungen erzeugt, so bedeutet dies eine Relativierung von Identität als scheinbar fixierter Position in der Gesellschaft. Identität ist nicht länger vorgegeben, sondern wird kontingent. ${ }^{57}$ Diese Kontingenzerfahrung ist typisch für die Moderne. Im Auflösungsprozess alter Ordnungen ergeben sich eine Vielzahl neuer Möglichkeiten, die ihrerseits wieder als Kontingenz erfahren werden. ${ }^{58}$

\subsection{Der Begriff der Ordnung}

Georg Simmels soziologische Analyse der Gesellschaft um die Jahrhundertwende macht die Dynamik, Komplexität und Kontingenz von Ordnung deutlich. Im Folgenden soll nun der Begriff der Ordnung selbst ins Zentrum gestellt werden, welcher für die europäische Geistesgeschichte von zentraler Bedeutung ist und die Geistesgeschichte seit den Vorsokratikern durch alle Epochen prägte. ${ }^{59}$ Mit Henri Bergson, vor allem aber mit Fritz Mauthner

56 Vgl. dazu auch aus soziologischer Warte: „Mit dem Begriff der ,Wechselwirkung“ ist das Prinzip einer Ordnung im Prozess genannt". Heinz Abels und Alexandra König, Sozialisation. Soziologische Antworten auf die Frage, wie wir werden, was wir sind, wie gesellschaftliche Ordnung möglich ist und wie Theorien der Gesellschaft und der Identität ineinanderspielen, Studientexte zur Soziologie (Wiesbaden: VS, Verlag für Sozialwissenschaften, 2010), 26. Hervorhebung i. O.

57 Zur Illustrierung dieses Punktes sei eine Stelle aus Theodor Fontanes Der Stechlin vorweggenommen, wo der durchaus liberal eingestellte Pastor Lorenzen im Gespräch mit Melusine sagt: „Der Hauptgegensatz alles Modernen gegen das Alte besteht darin, daß die Menschen nicht mehr durch ihre Geburt auf den von ihnen einzunehmenden Platz gestellt werden. Sie haben jetzt die Freiheit, ihre Fähigkeiten nach allen Seiten hin und auf jedem Gebiete zu bethätigen. Früher war man dreihundert Jahre lang ein Schloßherr oder ein Leinenweber; jetzt kann jeder Leinenweber eines Tages ein Schloßherr sein.“ (DS 264) Damit wird just jenes von Simmel anvisierte Spannungsverhältnis zwischen der Kleinstadt als vormoderne und der Großstadt als moderne Gruppe aufgenommen.

$5^{8}$ Vgl. dazu die Formulierung von Dillmann, dass „in der Kultur der Moderne [das] äußerst komplexe und teils aporetische Wechselverhältnis von Sinn, Notwendigkeit und Ordnung einerseits und Kontingenz und Unordnung andererseits" entstehe. Martin Dillmann, Poetologien der Kontingenz. Zufälligkeit und Möglichkeit im Diskursgefüge der Moderne, Kölner germanistische Studien (Köln: Böhlau, 2011), 37 f.

59 Für einen komprimierten Überblick zur Geschichte des Begriffs vgl. Meinhardt u. a., „Ordnung“. Im Lexikon Geschichtliche Grundbegriffe. Historisches Lexikon zur politischsozialen Sprache in Deutschland war laut Vorwort ein Artikel zu Ordnung geplant, auf den 
werden weitere Referenzgrößen der zeitgenössischen Reflexion des Ordnungsbegriffs der Moderne diskutiert sowie die Bedeutung des Konzepts von Ordnung für das Erzählen und deren Theoretisierung als Narratologie umrissen.

Für die Begriffsgeschichte von Ordnung in der Moderne stellen, analog zu derjenigen der Moderne allgemein, die Revolutionen im Übergang zum 19. Jahrhundert wichtige Umbrüche dar. Sie bilden „den Hintergrund für viele $\mathrm{O}$ [rdnungs] konzeptionen der Folgezeit, die sich nicht auf die Restauration der vergangenen $\mathrm{O}[\mathrm{rdnung}]$, sondern auf die Errichtung einer zukünftigen $\mathrm{O}$ [rdnung] richten. "60 Auch der anarchistischen Bewegung ging es nicht etwa darum, Ordnung als solche abzuschaffen, wie ihr vorgeworfen wurde. Vielmehr schwebten Proudhon, Bakunin und Kropotkin zukünftige, herrschaftsfreie Ordnungen der Freiheit vor. Damit wird auch dem wichtigen Umstand Rechnung getragen, dass die Definition dessen, was Ordnung und was Unordnung ist, immer auf eine Machtposition bezogen bleibt. ${ }^{61}$

aus Gründen der Erscheinungsreihenfolge der Teilbände verzichtet wurde. Im Vorwort zum vierten Band heißt es, dass der Artikel zu Ordnung in den Artikel zum Stichwort Verfassung im sechsten Band eingearbeitet wird, wo er entsprechend nur eingeschränkt diskutiert werden konnte. Damit ist deutlich gemacht, dass das Thema Ordnung eigentlich eine ausführlichere Behandlung verdient hätte. Vgl. Werner Conze, „Vorwort“, in Geschichtliche Grundbegriffe. Historisches Lexikon zur politisch-sozialen Sprache in Deutschland. Studienausgabe, hg. von Otto Brunner, Werner Conze und Reinhart Koselleck, Bd. 4, 8 Bde. (Stuttgart: Klett-Cotta, 2004), v-vi. Ebenfalls keinen Eintrag zu Ordnung verzeichnet: Anton Hügli und Poul Lübcke, Hrsg., Philosophielexikon. Personen und Begriffe der abendländischen Philosophie von der Antike bis zur Gegenwart, 6. Aufl. (Reinbek bei Hamburg: Rowohlt, 2005).

6o Meinhardt u. a., „Ordnung“, 1295.

61 In der Rede Die Ordnung (franz. 1881) widmet sich Peter Kropotkin dem Vorwurf der Unordnung und des Chaos, der an die anarchistische Bewegung herangetragen wurde. Zunächst macht er darauf aufmerksam, dass der Begriff Anarchie als verneinte Ordnung von den Feinden des Anarchismus als Spottruf verwendet wurde. Dann wendet er den Begriff ins Positive, indem er das angreift, was er als herrschende Ordnung definiert, die ausbeuterischen Machtverhältnisse: „Die Ordnung, das ist die Frau, die sich verkauft, um ihre Kinder ernähren zu können, das Kind, das sich entweder in eine Fabrik einsperren lassen oder Hungers sterben muß, der Arbeiter der zur Maschine erniedrigt wird. Es ist das drohende Gespenst des sich empörenden Arbeiters an den Türen der Reichen, des aufständischen Volkes an den Pforten der Herrschenden.“ Peter Krapotkin, „Die Ordnung [franz. 1881]“, in Worte eines Rebellen. Vom Verfasser genehmigte Uebersetzung aus dem Französischen; mit von ihm gutgeheißenen Fußnoten und Erläuterungen versehene Ausgabe, besorgt in dankbarem Angedenken, hg. \& übers. von Pierre Ramus, erste vollst., deutsche Ausgabe (Wien-Klosterneuburg: Verlag „Erkenntnis und Befreiung“, 1922), 70. Die Unordnung dagegen, die Anarchie, ist der Kampf gegen diese Ordnung mit dem Zweck der Etablierung einer neuen Ordnung, in der die „Harmonie, welche sich frei in den menschlichen Beziehungen entwickeln“ wird, verwirklicht ist. Krapotkin, 69. 


\subsubsection{Vitale Ordnung (Bergson)}

Henri Bergson, als Mitbegründer der Lebensphilosophie einer der einflussreichsten französischen Philosophen um die Jahrhundertwende, geht von der Faktizität von Ordnung aus: „Ordnung existiert, das ist Fakt.“62 Er versteht Ordnung als „eine gewisse Übereinkunft zwischen Subjekt und Objekt. Es ist der in den Dingen sich wiederfindende Geist.“ ${ }^{63}$ Bergson unterscheidet zwischen einer Ordnung des „Vitalen oder des Gewollten“ und einer „des Leblosen und des Automatischen“ ${ }^{64}$ Mit erstgenannter bezieht er sich auf Ordnungen, die nicht als solche auftreten und die kein ordnendes Prinzip aufzuweisen scheinen, wie in der Kunst, während letztere Ordnungen der Technik und der Physik angehören. Beide Ordnungstypen erfüllen jedoch dieselbe Funktion: „Eine wie die andere bewirkt, daß unsere Erfahrung sich wiederholt, eine wie die andere ermöglicht, daß unser Geist verallgemeinert."65 Welche dieser Ordnungen jeweils wirksam ist, ist kontingent und immer auf die jeweils andere, nicht aktivierte, bezogen. ${ }^{66}$ Entsprechend ist Unordnung immer bloß die Abwesenheit der Ordnungsform, die erwartet wird: „Unordnung [kann scheinbar] zwar durchaus die Negation einer Ordnung sein [...], doch [ist] diese Negation dann die implizite Feststellung der Anwesenheit der entgegengesetzten Ordnung“ ${ }^{67}$

Bergsons Konzept von Ordnung ist insofern interessant, als es eine Ordnung vorstellt, die keinem objektiven, mechanischen Regelwerk gehorcht, sondern viel eher der Intention eines Subjekts. Dies scheint gerade für prekäres Erzählen, das im Sinn der Performanz eine Ordnung erst bildet, fruchtbar. Gleichwohl ist auch diese Ordnung bei Bergson teleologisch gedacht, insofern als sich in der vitalen Ordnung die Absicht eines Geistes ausdrückt, die im Produkt wiederum eine statische Ordnung zu erzeugen scheint. Bergsons vitale Ordnung ist in einem allgemeinen Sinn auf Kunst bezogen, die, wie etwa seine Referenz Beethoven, keineswegs den Verfahren der Moderne um die Jahrhundertwende verwandt sind. ${ }^{68}$

Vgl. auch Ernst Zenker: „Es wird trotz der Anarchie oder eben ob derselben die höchste, natürliche Ordnung herrschen." Ernst Viktor Zenker, Der Anarchismus. Kritische Geschichte der anarchistischen Theorie (Jena: Verlag von Gustav Fischer, 1895), 56.

62 Henri Bergson, Schöpferische Evolution [franz. 1907], übers. von Margarethe Drewsen, Philosophische Bibliothek (Hamburg: Felix Meiner, 2013), 264.

63 Bergson, 255 .

64 Bergson, 256. Hervorhebung i. O.

65 Bergson, 257. Hervorhebung i. O.

66 Bergson, 256.

67 Bergson, $267 \mathrm{f}$.

68 Bergson, 256. 


\subsection{2 „Die subjektive Ordnung in unserem Kopfe“ (Mauthner)}

Henri Bergson stellte sich explizit in Opposition zu Immanuel Kant und dessen These von der Beschränktheit des menschlichen Erkenntnisvermögens. In seiner Kritik der reinen Vernunft (1781/1787) beschränkte Kant die Möglichkeiten des menschlichen Vorstellungsvermögens in den Formen der Anschauung von Zeit und Raum. Die Begrenzung des Erkenntnisvermögens, dargestellt an den „Antinomien der Vernunft", hat auch Konsequenzen für die Möglichkeit der Erkenntnis von Ordnung:

Die Ordnung und Regelmäßigkeit also an den Erscheinungen, die wir Natur nennen, bringen wir selbst hinein, und würden sie auch nicht darin finden können, hätten wir sie nicht, oder die Natur unseres Gemüts ursprünglich hineingelegt. Denn diese Natureinheit soll eine notwendige, d. i. a priori gewisse Einheit der Verknüpfung der Erscheinungen sein. ${ }^{69}$

Um die Jahrhundertwende verschärft die aufkommende Sprachphilosophie diese Erkenntniskritik. Die Grenzen der Erkenntnis werden nun innerhalb der Grenzen der Sprache verortet, die selbst zwar noch als logisch organisiert verstanden wird, in der Beziehung zwischen Signifikat und Signifikant jedoch arbiträr ist. ${ }^{70}$

In dieser erkenntniskritischen und sprachphilosophischen Denkschule steht Fritz Mauthner. ${ }^{71}$ Er ist der Ansicht, dass der Begriff der Ordnung „ein-

69 Immanuel Kant, „Kritik der reinen Vernunft“, in Immanuel Kant. Werkausgabe, hg. von Wilhelm Weischedel, Bd. 3, 12 Bde. (Frankfurt am Main: Suhrkamp, 1974), 179/ A 125. Hervorhebung i. O.

70 Ersteres behauptet Ludwig Wittgenstein im Tractatus logico-philosophicus, letzteres ist eine zentrale These in Ferdinand de Saussures linguistischem Modell der Sprache. Vgl. Ludwig Wittgenstein, „Tractatus logico-philosophicus“, in Ludwig Wittgenstein. Werkausgabe, Bd. 1: Tractatus logico-philosophicus. Tagebücher 1914-1916. Philosophische Untersuchungen (Frankfurt am Main: Suhrkamp, 1984), Abs. 5.5563; Ferdinand de Saussure, Grundfragen der allgemeinen Sprachwissenschaft, hg. von Charles Bally und Albert Sechehaye, 3. Aufl. (Berlin, Boston: De Gruyter, 2011).

71 Nicht zuletzt empfiehlt sich Mauthner durch seinen persönlichen und brieflichen Kontakt mit Fontane für die vorliegende Untersuchung. Regelmäßig verfassten sie Rezensionen zu den Werken des jeweils anderen. Fontane hat sich in Briefen gegenüber anderen Briefpartnern durchaus abfällig über Mauthner geäußert, was diesen beim posthumen Erscheinen der Briefe nachhaltig verletzt hat. Helmuth Nürnberger beschreibt die Beziehung der beiden als einseitig: „Für Fontane handelt es sich bei der Beziehung zu Mauthner um einen der zahlreichen Kontakte, die der alte Schriftsteller zu Persönlichkeiten des damaligen literarischen Berlin hauptsächlich aus beruflichem Interesse pflegte. [...] Wichtiger als für Fontane sind die Kontakte zwischen den beiden Autoren für den dreißig Jahre jüngeren Mauthner gewesen, wobei weniger äußere als innere Rücksichten ins Spiel kommen. Das gilt auch für die Zeit nach Fontanes Tod, als Mauthner den 
zig und allein der verbalen Welt angehört, der Welt der Apperzeption oder des Denkens".72 Die Sprache macht die Welt erst erfahrbar und begreifbar, da sie "die Welt vorläufig zu klassifizieren oder zu ordnen versucht hat." ${ }^{43}$ Dieses vorläufige "System von Begriffen“ ist jedoch nur "heuristisch zweckmäßig“. Denn dass beim „Entstehen der Dinge [...] irgendeine Ordnung, noch irgendein System mitgewirkt haben könnte" sei abwegig, ,weil nur der Zweck ordnet, der Menschenzweck, Zweck aber der Natur fremd ist, welche nur Ursachen kennt ${ }^{\text {" }} .74$

Ausführlicher als im Wörterbuch der Philosophie (1910) bezieht Mauthner in seinen Beiträgen zur Kritik der Sprache (1901/1902), einem grundlegenden Werk der Sprachphilosophie wie der Sprachkrise, das Ordnungsproblem auf die veränderte Wahrnehmung in der Großstadt: Die „Ordnung, welche der Menschengeist in die Wirklichkeitswelt hineinverlegt, [ist] nichts Anderes [...] als diese Aufmerksamkeit meiner Sinne auf das Farbenzeichen meiner Straßenbahn. “75 Damit meint Mauthner, dass die wahrgenommene Ordnung, also die Möglichkeiten der Orientierung von der Aufmerksamkeit der Sinne abhängt, die wiederum von Umständen, wie Tag oder Nacht, Stadt oder Land und der seelischen Verfassung beeinflusst ist. Die Ordnung, die man im großstädtischen Alltag erlebe, sei nichts anderes als unsere Erfahrung des Zurechtfindens. In Wirklichkeit herrsche ein grenzenloses und zusammenhangsloses Chaos.

Die scheinbare Zweckmäßigkeit der Welt ist doch nur unser egoistisches Zurechtfinden in dem regelmäßigen Chaos der Wirklichkeit, und die Regelmäßigkeit

postumen Briefpublikationen entnehmen mußte, daß Fontanes Urteil über ihn wesentlich kritischer war, als er geglaubt hatte. Für sein labiles Selbstgefühl bedeutete dies keine geringe Erschütterung.“ Helmuth Nürnberger, „Fontane und Mauthner“, in Literarisches Doppelportrait. Theodor Fontane / Fritz Mauthner, hg. von Uta Kutter (Stuttgart, Marbach: Verein der Freunde der Akademie für Gesprochenes Wort, 200o), 82f. Nürnberger sieht darin gerade ein typisches Beispiel für Fontanes „berühmt-berüchtigte Ambiguität bzw. ,Ambivalenz', das Schwankende und Mehrdeutige seiner Urteile und Stellungnahmen“. Nürnberger, 83 f. Fritz Mauthners Beiträge zu Fontane sind erhalten im Band: Uta Kutter, Hrsg., Literarisches Doppelportrait Theodor Fontane / Fritz Mauthner, Schriften der Akademie für Gesprochenes Wort (Stuttgart, Marbach:Verein der Freunde der Akademie für Gesprochenes Wort, 2000).

72 Fritz Mauthner, Wörterbuch der Philosophie. Neue Beiträge zu einer Kritik der Sprache, Bd. 2 (München, Leipzig: Georg Müller, 1910), 220.

73 Mauthner, Bd. 2: 220. Hervorhebung i. O.

74 Mauthner, Bd. 2: 221. Hervorhebung i. O.

75 Fritz Mauthner, Beiträge zu einer Kritik der Sprache [19o1/o2], 2. Aufl., Bd. 3:Zur Grammatik und Logik (Stuttgart, Berlin: J.G. Cotta'sche Buchhandlung Nachfolger, 1913), 588. Hervorhebung i. O. 
dieses Chaos ist doch nur die Wiederkehr der unzähligen Egoismen, die als Zellen, als Individuen und als Gruppen oder Aktiengesellschaften einzig und allein selbst leben wollen. ${ }^{76}$

Dementsprechend gibt es keine „Weltordnung“, ${ }^{77}$ oder wenn es sie gäbe, so würde der Mensch sie nicht kennen.

Wir wissen nur von der subjektiven Ordnung in unserem Kopfe; wir wissen nicht, ob wir das, was dieser Ordnung objektiv entspricht, noch unter der Menschenvorstellung Ordnung begreifen können. [...] Der Mensch hat die Ordnung in die Natur hineingetragen, durch seine arme Sprache. Nachher verzweifelt er, wenn er seine Ordnung in der Natur nicht finden kann. ${ }^{78}$

Mauthners Konzeptualisierung der Ordnung als etwas Künstliches, das zutiefst kontingent und auf das Erleben des Menschen selbst bezogen ist, ist grundlegend für die Moderne. In dieser Reduktion ist sie gleichsam ein gemeinsamer Nenner zahlreicher modernistischer Diskurse wie Diskursen über die Moderne.

\subsubsection{Ordnung in Rhetorik und Narratologie}

In den Literaturwissenschaften ist die Kategorie der Ordnung zunächst in der Rhetorik wichtig. Ausschließlich unter diesem Aspekt wird Ordnung denn auch im Reallexikon der Literaturwissenschaften behandelt, das einzig das Begriffspaar ordo artificialis/ordo naturalis ausweist. Damit werden die zwei "Ordnungsprinzipien“79 in der dispositio der Rede bezeichnet, die sowohl in der Abfolge der Untereinheiten als auch in der Gestaltung der Untereinheiten selbst, insbesondere der narratio, Anwendung finden. Entspricht dem ordo naturalis in der antiken Rhetorik die Abfolge von exordium (Einleitung) narratio (Erzählung des Falles) - argumentatio - conclusio, so sammelt die Bezeichnung ordo artificialis ,jede Abweichung davon“. 80 Diese Unterscheidung lässt sich auf die Poetik beziehen, wenn die Rede im ordo naturalis die Ereignisse in der tatsächlichen, chronologischen Reihenfolge wiedergibt. Der ordo artificialis weicht von dieser Reihenfolge ab und nimmt Umstellungen

76 Mauthner, Wörterbuch der Philosophie. Neue Beiträge zu einer Kritik der Sprache, Bd. 2: 220.

77 Mauthner, Beiträge zu einer Kritik der Sprache [1901/o2], Bd. 3: Zur Grammatik und Logik: 59 o.

78 Mauthner, Bd. 3: Zur Grammatik und Logik: 59o.

79 Fritz Peter Knapp, „Ordo artificialis/Ordo naturalis“, in Reallexikon der deutschen Literaturwissenschaft. Neubearbeitung des Reallexikons der deutschen Literaturgeschichte gemeinsam mit Harald Fricke, Klaus Grubmüller und Jan-Dirk Müller. Bd. 2, hg. von Harald Fricke (Berlin, New York: De Gruyter, 2007), 766. 
vor, setzt Vor- und Rückblenden in der Zeitstruktur ein, so dass die Ereignisse nicht mehr in der Reihenfolge erzählt werden, in der sie stattfanden.

Mit dieser Übertragung ist der Bezug zu narratologischen Analysekategorien schon vollzogen. Bei Gérard Genette betrifft die Kategorie der Ordnung ausschließlich das Verhältnis der Zeitabfolge der erzählten Geschichte zu der Abfolge des Erzählens. Entsprechend ergeben sich Möglichkeiten eines zeitdeckenden, eines proleptischen und eines analeptischen Erzählens. Genette räumt in seiner Systematik auch der „Macht der Erzählung, sich von den Zwängen derchronologischen Reihenfolge der erzählten Geschichte vollständig zu befreien“ und "achronische Strukturen“" zu erzeugen, Platz ein. ${ }^{81}$ Als alternative Ordnungsverfahren zur Zeit, Ereignisse „zu gruppieren “82, führt Genette die räumliche Kontiguität, klimatische Motive und thematische Ebenen an, die der Erzählung eine „temporale Autonomie “83 ermöglichen. Gerald Princes Nomenklatur, die im angelsächsischen Raum für die Narratologie so einflussreich ist wie es im deutsch- und französischsprachigen Genettes Klassifikation ist, sieht in seinem Dictionary of Narratology unter dem Eintrag „order“ ebenfalls einzig die zeitliche Relation vor: „The set of relations between the order in which events (are said to) occur and the order in which they are recounted. ${ }^{44}$

Auf einer höheren Ebene, aus Sicht der Semiotik, lässt sich auch die Erzählung selbst als eine auf spezifische Art und Weise geordnete Menge von Zeichen beschreiben. Die Narratologie versucht, diese Ordnung zu analysieren und zu beschreiben. ${ }^{85}$ Wird im Folgenden von Erzählordnung gesprochen, ist in der Regel nicht nur die zeitliche Abfolge von Ereignissen einer Erzählung gemeint, sondern das Erzählmodell in seiner Teilhabe an Ordnungsmodellen auf unterschiedlichen Ebenen: Vom Kommunikationsmodell mit variierenden diegetischen Ebenen, über semantische Ordnungen bis zur Ordentlichkeit von Beschreibungen und Syntax. Auf diese Weise soll der Begriff der Ordnung im Erzählen für weitere Verwendungsweisen fruchtbar gemacht werden, referiert doch eine Erzählung in vielfältiger Weise auf Ordnungen, wie sie auch vielfältige Ordnungen in sich zusammenführt.

Die Narratologie kann gerade mit Gérard Genette, Gerald Prince und Seymour Chatman, aber auch mit Algirdas Julien Greimas' Aktantentheorie,

81 Genette, Die Erzählung, 51. Hervorhebung i. O.

82 Genette, 51. Genette verzichtet auf das Verb „ordnen“ und wählt stattdessen "gruppieren“, um die Kategorie der Ordnung der Zeitstruktur vorbehalten zu können.

83 Genette, 52. Hervorhebung i. O.

84 Gerald Prince, A Dictionary of Narratology (Lincoln, London: University of Nebraska Press, 1987), 68.

85 Diese Beschreibung gilt streng genommen nur für Modelle, die von einer Textstruktur ausgehen, die universell narrativ ist. Kognitive Modelle dagegen betonen, dass die Eigenschaft eines Textes, Erzählung zu sein, erst im Lektüreprozess aufscheint. 
Claude Brémond, Tzvetan Todorov und anderen schon in der Blüte der strukturalistischen Theoriebildung in den 196oer-Jahren als Wissenschaft des Ordnens bezeichnet werden. Auch zeitgenössische Erzähltheorien, klassische wie bei Wolf Schmid, die sich stark am Strukturalismus anlehnen, oder postklassische wie bei Monika Fludernik, zeichnen sich gemeinhin durch einen hohen Grad an Systematikaus. ${ }^{86}$ Nicht zuletzt ist auch die Narratologie Teiljener großen, oben nachgezeichneten Ordnungswelle um die Jahrhundertwende. ${ }^{87}$

Diese Systeme narratologischer Typisierung lassen, so feinmaschig sie auch sind, zwangsläufig Lücken offen, in denen sich moderne und postmoderne Texte gleichsam festsetzen können. Die jüngere Entwicklung der Narratologie hat diesem Umstand insofern Rechnung getragen, als neue Ordnungskriterien erstellt und intermediale Formen mitberücksichtigt werden. Die postklassische Narratologie durchbricht mit diversen Ansätzen die Vorstellung eines zumindest in den Grundsätzen geeinten, verbindlichen narratologischen Systems. ${ }^{88}$

In den am Erzählen interessierten Kulturwissenschaften hat der Ordnungsbegriff ebenfalls eine besondere Bedeutung. Erzählen wird als Ordnungsstrategie aufgefasst, die die Funktion der Sinnerzeugung erfüllt. Das Paradigma der Kulturwissenschaften lautet, dass Erzählungen „in unterschiedlichen kulturellen Kontexten auf grundlegende Weise zur Organisation von menschlicher Erfahrung bei[tragen], indem sie das mehr oder minder kontingente Geschehen in der Lebenswelt des Menschen in kohärente Ordnungen überführen". 89

86 Zum Begriff der klassischen und postklassischen Narratologie vgl. Jan Alber und Monika Fludernik, Hrsg., Postclassical Narratology. Approaches and Analyses, Theory and Interpretation of Narrative (Columbus: Ohio State University Press, 2010).

87 Die Gründung der modernen Narratologie ist auf die Zeit der Jahrhundertwende anzulegen, wenn auch der Begriff „Narratologie“ bzw. französisch „narratologie“ erst 1969 von Tzvetan Todorov geprägt wurde. Im englischsprachigen Raum wird der Beginn der modernen Narratologie gewöhnlich mit Henry James' Essay The Art of Fiction markiert, der 1884 im Longman's Magazine erschien. Im deutschsprachigen Raum ist es Käte Friedemanns Die Stellung des Erzählers in der epischen Dichtung (1910). Auch Vladimir Propps Morphologie des Märchens, ein großangelegter Ordnungsversuch des Märchens, ist hier zu erwähnen (russ. 1928).

88 Vgl. Ansgar Nünning, „Narratology or Narratologies? Taking Stock of Recent Developments, Critique and Modest Proposals for Future Usages of the Term“, in What is Narratology? Questions and Answers Regarding the Status of a Theory, hg. von Tom Kindt und Hans-Harald Müller, Narratologia. Contributions to Narrative Theory 1 (Berlin, Boston: De Gruyter, 2003), 239-275.

89 Die ausführliche Textstelle lautet: „Sieht man im Erzählen in der skizzierten Art und Weise [d. h. im Sinn Paul Ricoeurs als ,Refiguration' oder als Hayden Whites ,Emplotment'; L. G.] in erster Linie die ,Herstellung von Zusammenhang aus Einzelheiten` in Gestalt 
Es soll hier gerade darum gehen, Erzählen als Kategorie zu begreifen, die nicht nur Kohärenz, sondern auch Ambivalenz herstellen will. Diese Studie positioniert sich so zwischen dem ",ambiguitätsvergessene[n] 'Blick der neueren kulturwissenschaftlichen Forschung“ und dem ",ambiguitätsversessene[n] “ Blick dekonstruktiver Lektüren. ${ }^{90}$

Für diese Analysen ist der Begriff der Ambivalenz zentral, der häufig wenig spezifisch im Sinn von Zwiespältigkeit, Vieldeutigkeit und Unsicherheit gebraucht wird. ${ }^{91}$ Er wird verwendet, als seien er und die ihm naheliegenden Konzepte der Ambiguität und Amphibolie „keine klärungsbedürftigen Be-

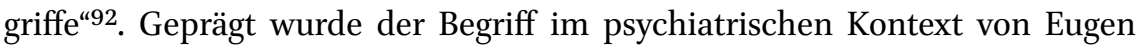
Bleuler als eine der "Störungen der Affektivität“, die in der Gleichwertigkeit zweier gegensätzlicher Gefühlsregungen besteht. Während auch der „Normale $[\ldots]$ zwei Seelen in seiner Brust“ fühle, so könne er aus den „ambivalente $[n] \mathrm{Ge}-$ fühlsbetonungen“ dennoch ein „Fazit“" ziehen. ${ }^{93}$ Anders der pathologische Fall: „Der Kranke aber kann oft die beiden Strebungen nicht zusammenbringen; er haßt und liebt nebeneinander, ohne daß sich die beiden Affekte abschwächten oder überhaupt beeinflußten. ${ }^{\text {"94 }}$

von Geschichten, so tragen Narrationen in unterschiedlichen kulturellen Kontexten auf grundlegende Weise zur Organisation von menschlicher Erfahrung bei, indem sie das mehr oder minder kontingente Geschehen in der Lebenswelt des Menschen in kohärente Ordnungen überführen. Nicht zuletzt dank dieses Verständnisses von Erzählen als ,Ordnen` und ,Erklären` konnte Narrativität in den letzten Jahren zu einem Paradigma der Kulturwissenschaften avancieren.“ Abel, Blödorn und Scheffel, „Ambivalenz und Kohärenz", if.

$90 \quad$ Abel, Blödorn und Scheffel, 2f.: „Der eigentlichen Komplexität von Erzählungen und der mit ihnen verbundenen Ordnungs- und Sinnbildungsprozesse scheint uns weder der ,ambiguitätsversessene‘ Blick unterschiedlicher Spielarten der Dekonstruktion noch der ,ambiguitätsvergessene‘ Blick der neueren kulturwissenschaftlichen Forschung zu entsprechen." Vgl. dazu auch die von Koschorke hervorgehobene Problematik, die sich aus einer Ausdehnung des Erzählbegriffs auf Erkenntnis und Wissenschaftlichkeit in den Kulturwissenschaften ergibt. Albrecht Koschorke, Wahrheit und Erfindung. Grundzüge einer allgemeinen Erzähltheorie (Frankfurt am Main: Fischer, 2012), 329-340.

91 Kurt Lüscher, „Robert Walsers Sensibilität für Ambivalenzen. Eine transdisziplinäre Annäherung“, in Robert Walsers Ambivalenzen, hg. von Kurt Lüscher u. a., 2., durchgesehene und korrigierte Aufl., Robert Walser-Studien 3 (Paderborn: Wilhelm Fink, 2019), 10.

92 Frauke Berndt und Stephan Kammer, „Amphibolie - Ambiguität - Ambivalenz. Die Struktur antagonistisch-gleichzeitiger Zweiwertigkeit“, in Amphibolie - Ambiguität Ambivalenz, hg. von Frauke Berndt und Stephan Kammer (Würzburg: Königshausen \& Neumann, 2009), 8.

93 Eugen Bleuler, Lehrbuch der Psychiatrie (Berlin: Julius Springer, 1916), 92. Hervorhebung i. O.

94 Bleuler, 92. 
Vom psychiatrischen Kontext anfangs des 20. Jahrhunderts abstrahiert und für die Zwecke dieser Studie fruchtbar gemacht, lässt sich Ambivalenz mit Zygmunt Bauman, wie oben gesehen, definieren als „die Möglichkeit, einen Gegenstand oder ein Ereignis mehr als nur einer Kategorie zuzuordnen“. ${ }^{95}$ Wie bei Bleuler hat Ambivalenz „Unentschieden zur Folge“ weshalb sie „als Unordnung" erfahren wird. ${ }^{96}$ Im Konflikt verschiedener Alternativen impliziert Ambivalenz „eine Denkfigur des ,Sowohl-als-auch', [...] eine Dialektik, die nicht in eine Synthese mündet. ${ }^{47}$ In diesem Zustand der Ambivalenz sind "gleichwertige, gleichzeitige, aber zueinander antagonistische Skripte“ wirksam. ${ }^{98}$ Für Ambivalenz sind der "Handlungsverzicht, das Zaudern, Schwanken und Aufschieben von Entscheidungen“ in diesem vielfältigen Handlungs- und Deutungsangebot zentral. ${ }^{99}$

Ambivalenz kann sich sowohl auf die narrative Struktur eines Textes wie auf dessen übergeordneten Sinn, auf die „narrative Detailstruktur (Mikroebene), aber auch [auf] die Gesamtbedeutung der Narration (Makroebene)“ beziehen. ${ }^{100}$ Dies geschieht mit verschiedenen narrativen Verfahren, wie „Vagheit (Unbestimmtheit), Ambiguität (Mehrdeutigkeit) und Brüche[n], aber auch verschiedene, miteinander konkurrierende Erklärungsmuster innerhalb einer Erzählung" sind zur Ambivalenz dazuzurechnen. ${ }^{101}$

Die vorliegende Studie greift auf dieses Bedeutungsspektrum des Ambivalenzbegriffs zurück, um eine große Vielfalt an ambivalenten Strukturen, sowohl auf der Ebene der Semantik, der erzählten Geschichte, als auch des Erzählens selbst, bei den drei Autoren Fontane, Walser und Kafka herausarbeiten zu können.

Für die weitere Analyse wird es hilfreich sein, die Begriffe der Kontingenz und Ambivalenz mittels der basalen narratologischen Unterscheidung von

95 Bauman, Moderne und Ambivalenz, 13.

96 Bauman, 13 .

97 Lüscher, „Robert Walsers Sensibilität für Ambivalenzen. Eine transdisziplinäre Annäherung", 15 .

98 Stephan Kammer, „Krisis‘ (1928/1929). Robert Walsers Ambivalenznarrativ“, in Robert Walsers Ambivalenzen, hg. von Kurt Lüscher u. a., 2., durchgesehene und korrigierte Aufl., Robert Walser-Studien 3 (Paderborn: Wilhelm Fink, 2019), 180.

99 Kammer, 181. Interessant ist hier auch der Aspekt des Krisennarrativs, wie ihn Stephan Kammer herausarbeitet: Der Aufschub des Entschlusses korrespondiert mit der „in Krisengegenwarten gebotene[n] Ablösung von Handlung durch Erzählungen." Kammer, 181. Kammer zeigt in seiner Lektüre von Robert Walsers spätem Prosatext Krisis, wie dieser den verbreiteten zeitgenössischen Diskurs um die Krise aufnimmt und in seiner Ironie äußerst ambivalent darstellt.

100 Abel, Blödorn und Scheffel, „Ambivalenz und Kohärenz“, 5.

101 Abel, Blödorn und Scheffel, 5. Hervorhebung i. O. 
story und discourse zu präzisieren. Kontingenz lässt sich auf der Ebene der story verorten. Sie wird von den Figuren erlebt und steht im Gegensatz zur Notwendigkeit bzw. der Selbstverständlichkeit eines Geschehnisses, also dem ordnungsgemäßen Ablauf. Wenn etwa in Fontanes Stechlin der konservative Woldemar die ebenfalls konservative Armgard der progressiven Melusine vorzieht, so ist dies vor dem Hintergrund der Romanhandlung naheliegend und bleibt also im Rahmen der erwarteten Ordnung. Der Erzähler kann dabei die Kontingenz der Ereignisse mehr oder weniger stark durchscheinen lassen. Auf der Ebene der Beschreibung und Bewertung, im discourse, operiert die Ambivalenz. Dies gilt sowohl für die Figuren, die ein Ereignis in seiner Bedeutung ambivalent beschreiben können - etwa die Diskussion über die Bedeutung der Textilfärberei „Spindlersfelde“ für die „Damen“ -, als auch für den Erzähler des Ereignisses: Ein Ereignis oder ein Diskurs wird aus verschiedenen Perspektiven erzählt und unterschiedlich kommentiert, ohne dass die Erzählung eine Haltung favorisiert. Auch die Erzählung in ihrer Gesamtheit nimmt durch die Verfahren, mittels denen sie Meinungen darstellt, Positionen ein. Sie kann eine Selbstverständlichkeit subtil als kontingent darstellen oder einen Zufall als notwendiges Ereignis im Rahmen einer übergeordneten Kausalität. Bei Fontane gilt dies besonders für die oben genannte Heirat zwischen Woldemar und Armgard, die, wie später deutlich wird, trotz Kontingenz - auch Melusine ist wählbar - gerade der Ordnung wegen geschehen muss. Die Selbstverständlichkeit mit der Woldemar sich gleichwohl für Armgard entscheidet, die nicht aus der story motiviert ist, lässt sich als Konstruktion von Ordnung lesen, die gerade in ihrer Kontingenz eine Ambivalenz offenbart, die die Ordnung wiederum relativiert und schwächt. So wird deutlich, dass die Begriffe der Kontingenz, Ambivalenz, Notwendigkeit und Ordnung in einem komplexen Beziehungsnetz zueinanderstehen.

\section{$1.4 \quad$ Vorgehen}

Was die oben diskutierten Positionen von Bauman, Simmel, Bergson und Mauthner sichtbar machen, ist, dass Ordnung in der Moderne erst hergestellt werden muss und als Konstruktion kontingent bleibt. Um die Jahrhundertwende hat sich sowohl die gesellschaftliche Ordnung, wie auch die Beschreibung derselben kompliziert. Mit dieser Komplizierung geht eine Unsicherheit einher, die den prekären Status der Ordnung spiegelt.

Für die Literatur lässt sich der Ordnungsbegriff in zweierlei Hinsicht fruchtbar machen. Zum einen für die Analyse der Darstellung von Ordnung auf der Inhaltsebene, also die soziale, politische, technologische, topografische 
Semantik. Zum anderen lassen sich die Verfahren der Herstellung narrativer Ordnung untersuchen, die ihrerseits eine gewisse Ordnung aufweisen und sich zu anderen Ordnungen in Bezug setzen. Dies wurde eingangs mit der Lektüre von Robert Walsers Simon. Eine Liebesgeschichte skizziert. Gerade die für diese Arbeit zentralen Autoren Walser, Franz Kafka und Theodor Fontane haben ihre je eigenen Methoden, wie sie Ordnung herstellen, konzeptualisieren und unterwandern. Im prekären Erzählen etablieren sie prototypische Strategien in Darstellung und Umgang mit dem Ordnungsproblem der Moderne.

Der Begriff der Ordnung wird hier also auf verschiedenen Ebenen verwendet und diskutiert: Als diskursiver Kontext der Autoren, als dargestellte Ordnung auf der Inhaltsebene, als in dieser Darstellung evaluierte und konzeptualisierte Ordnung und insbesondere als Ordnungsverfahren im Erzählen, das wiederum Erzählordnungen aufweist, thematisiert und zitiert. Die lange Geschichte des Begriffs und seine Bedeutungsvielfalt erfordern eine dezidierte Beschränkung der Diskussion desselben auf die Zwecke dieser Studie: Die vorliegende Studie leistet eine Bearbeitung des narrativen Ordnungsproblems bei Fontane, Kafka und insbesondere Walser und konturiert damit den Begriff des prekären Erzählens. Dieses Konzept des prekären Erzählens ermöglicht einen differenzierten Blick auf die Spezifika der genannten Autoren und Texte, ihren Bezug zur Ordnung sowie ihr Verhältnis zur Epoche vor dem Hintergrund des Ordnungsproblems. Vor allem stellt der Begriff des prekären Erzählens ein Konzept zur Debatte, das Erzählformen, die gemeinhin durch Negationen als defizitär beschrieben werden, positiv als Ordnungsleistungen bestimmen kann. Diese Studie wirft ein neues Licht auf die narrative Herstellung und Thematisierung von Ordnungen, entwickelt daraus den Begriff des prekären Erzählens und stellt so auch überraschende Bezüge zwischen den Autoren und ihrem Zeitkontext her.

In dieser Einleitung wurde auf die Problematik des prekären Erzählens in der Moderne aufmerksam gemacht. Das methodische Vorgehen wurde dargelegt und die Verwendung des Ordnungsbegriffs geklärt. Der Begriff der Ordnung wurde konturiert und das Ordnungsproblem der Moderne diskutiert. Diese Vorarbeit erlaubt es nun im Folgenden, textnah zu argumentieren und die Beziehung von Ordnungsbildung und Unordnung als prekäres Erzählen zu analysieren.

Im Zentrum des zweiten Kapitels steht die narrative Kontrolle der Ambivalenz in Theodor Fontanes Alterswerk Der Stechlin. Im Stechlin wird die Ambivalenz der Moderne in engen, narrativen Bahnen geführt. Trotz aller Kontrolle ist sie als Motor für die Entfaltung der Geschichte unentbehrlich. Fontanes Strategie im Umgang mit dem Ordnungsproblem ist ein überdeutliches Verstecken, Lenken und Kontrollieren der, mit Bauman, in ihrem Wesen 
jedoch unkontrollierbaren Ambivalenz. Mit vielfältigen semantischen und narrativen Verfahren zeigt Der Stechlin eine Welt, deren Ordnung zerfällt und nur von der Erzählung selbst noch zusammengehalten werden kann, die in dieser Künstlichkeit jedoch prekär ist. Vor diesem Hintergrund werden die Texte Robert Walsers und Franz Kafkas in ihrer Ausrichtung deutlich als vielfach gebrochene und widersprechende Fortführungen Fontanes lesbar.

Das dritte Kapitel ist Robert Walser gewidmet, der deshalb den Schwerpunkt dieser Studie bildet, weil sich bei ihm das prekäre Erzählen besonders deutlich und vielfach reflektiert als Performanz zeigt. Die Performanz stellt die Basis für zahlreiche, herkömmliche Formen des Erzählens unterwandernde Erzählverfahren als ortlose und häufig paradoxe Herkunft des Erzählens. Drei Verfahren des prekären Erzählens stehen bei Walser im Zentrum: Insbesondere im Frühwerk wird explizit die Grenze von Erzählung und Bild verhandelt. In der Walser-Forschung bislang am ausführlichsten diskutiert wurde das Verfahren der Digression, die hier hauptsächlich an Der Spaziergang und am „Räuber“Roman sowie an zwei späten Prosastücken diskutiert wird. Sichtbar werden dabei auch Verbindungslinien vom Spaziergang zum "Räuber"-Roman in der Radikalisierung der Spaziergänger-Figur wie der Erzähler-Spaltung. Schließlich wird Walsers Strategie des performativen Erzählens analysiert, das den Akt des Erzählens als Ursache der Erzählung selbst vorstellt und damit einen zentralen Aspekt des prekären Erzählens betont. Das Kapitel findet seinen Abschluss in einer Analyse der Figurationen des Erzählens, die die prekären Erzählverfahren in der Metapher des Essens fassen. Fern davon, Vollständigkeit anzustreben, ist das Textkorpus bei Walser umfangreicher als bei Fontane und Kafka und bildet alle Werkphasen ab.

Auf der Basis der Analyse der Texte Robert Walsers wird im vierten Kapitel Franz Kafkas ,Bau-Konvolut' als eine geradezu gegensätzliche Umgangs- und Ausdrucksweise mit dem Ordnungsproblem der Moderne lesbar. Diese besteht insbesondere in einer Konzentration des Problems auf inhaltlicher Ebene, auf einer Destabilisierung der Semantik, einer Prekarisierung der Erzählordnung durch alogische Zeit- und hintergründige Raumkonstruktion, wie einer logisch unmöglichen Erzählstimme. In der narrativen Tendenz zur Performanz im Bau wird auch eine deutliche Parallele zu Walser sichtbar.

Auf die Analyse der narrativen Verfahren zur Herstellung von Ordnung in den textzentrierten Kapiteln folgt im fünften Kapitel eine Auseinandersetzung mit zeitgenössischer narratologischer Theoriebildung. Mit Marie-Laure Ryans modes of narrativity, Monika Fluderniks, natural' narratology, dem Konzept der unnatural narratology sowie einer Diskussion der antinarratives wird eine theoretische Rahmung und Differenzierung des prekären Erzählens erzielt. 